\title{
Regular Path Expressions in Feature Logic
}

\author{
Rolf Backofen
}

May 1993

Deutsches Forschungszentrum für Künstliche Intelligenz $\mathrm{GmbH}$

Postfach 2080

67608 Kaiserslautern, FRG

Tel.: + 49 (631) 205-3211

Fax: + 49 (631) 205-3210
Stuhlsatzenhausweg 3

66123 Saarbrücken, FRG

Tel.: + 49 (681) 302-5252

Fax: + 49 (681) 302-5341 


\section{Deutsches Forschungszentrum für Künstliche Intelligenz}

The German Research Center for Artificial Intelligence (Deutsches Forschungszentrum für Künstliche Intelligenz, DFKI) with sites in Kaiserslautern and Saarbrücken is a non-profit organization which was founded in 1988. The shareholder companies are Atlas Elektronik, Daimler-Benz, Fraunhofer Gesellschaft, GMD, IBM, Insiders, Mannesmann-Kienzle, Sema Group, Siemens and Siemens-Nixdorf. Research projects conducted at the DFKI are funded by the German Ministry for Research and Technology, by the shareholder companies, or by other industrial contracts.

The DFKI conducts application-oriented basic research in the field of artificial intelligence and other related subfields of computer science. The overall goal is to construct systems with technical knowledge and common sense which - by using Al methods - implement a problem solution for a selected application area. Currently, there are the following research areas at the DFKI:

$\square$ Intelligent Engineering Systems

$\square$ Intelligent User Interfaces

$\square$ Computer Linguistics

$\square$ Programming Systems

$\square$ Deduction and Multiagent Systems

$\square$ Document Analysis and Office Automation.

The DFKI strives at making its research results available to the scientific community. There exist many contacts to domestic and foreign research institutions, both in academy and industry. The DFKI hosts technology transfer workshops for shareholders and other interested groups in order to inform about the current state of research.

From its beginning, the DFKI has provided an attractive working environment for Al researchers from Germany and from all over the world. The goal is to have a staff of about 100 researchers at the end of the building-up phase.

Friedrich J. Wendl

Director 
Regular Path Expressions in Feature Logic

Rolf Backofen

DFKI-RR-93-17 
Parts of this report have been published in the Proceedings of the Fifth International Conference on Rewriting Techniques and Applications, and in the Proceedings of the 31st Annual Meeting of the Association for Computational Linguistics

This work has been supported by a grant from The Federal Ministry for Research and Technology (FKZ ITWM-9002 0).

(C) Deutsches Forschungszentrum für Künstliche Intelligenz 1993

This work may not be copied or reproduced in whole of part for any commercial purpose. Permission to copy in whole or part without payment of fee is granted for nonprofit educational and research purposes provided that all such whole or partial copies include the following: a notice that such copying is by permission of the Deutsche Forschungszentrum für Künstliche Intelligenz, Kaiserslautern, Federal Republic of Germany; an acknowledgement of the authors and individual contributors to the work; all applicable portions of this copyright notice. Copying, reproducing, or republishing for any other purpose shall require a licence with payment of fee to Deutsches Forschungszentrum für Künstliche Intelligenz. 


\title{
Regular Path Expressions in Feature Logic
}

\author{
Rolf Backofen \\ Deutsches Forschungszentrum für Künstliche Intelligenz \\ Saarbrücken \\ backofen@dfki.uni-sb.de
}

\begin{abstract}
We examine the existential fragment of a feature logic, which is extended by regular path expressions. A regular path expression is a subterm relation, where the allowed paths for the subterms are restricted to any given regular language. We will prove that satisfiability is decidable. This is achieved by setting up a quasiterminating rule system.
\end{abstract}




\section{Contents}

1 Introduction 3

2 The Method 4

$\begin{array}{lll}3 & \text { Preliminaries } & 7\end{array}$

4 Feature Trees $\quad 10$

5 Prime, Pre-Solved and Solved Clauses 11

6 The First Phase 14

6.1 A Set of Rules . . . . . . . . . . . . . . . . 14

6.2 Some Properties of the Rule System . . . . . . . . . . 19

6.3 Soundness and Completeness . . . . . . . . . . . . 22

6.4 Quasi-Termination .................... 29

7 The Second Phase: Satisfiability of Pre-Solved Clauses $\quad 30$

8 Conclusion $\quad 34$ 


\section{Introduction}

Feature descriptions are used as the main data structure of so-called unification grammars, which are currently a popular family of declarative formalisms for processing natural language [Shi86]. More recently, feature descriptions have been proposed as a constraint system for logic programming [AKN86, AKLN87, AKP91, AKPS92b, ST92]. They provide for a partial description of abstract objects by means of functional attributes called features. As an example consider the feature description (in matrix notation):

$$
x: \exists y\left[\begin{array}{ll}
\text { woman } & \\
\text { father } & :\left[\begin{array}{l}
\text { engineer } \\
\text { age }: y
\end{array}\right] \\
\text { husband } & :\left[\begin{array}{l}
\text { painter } \\
\text { age }: y
\end{array}\right]
\end{array}\right],
$$

which may be read as saying that $x$ is a woman whose father is an engineer, whose husband is a painter and whose father and husband are both of the same age.

Feature description have been proposed in various forms with various formalizations [AK86, KR86, RK86, Joh88, Smo92, Joh91]. We will follow the logical approach introduced by Smolka [Smo92], where feature descriptions are standard first order formulae interpreted in first order structures. In this formalization features are considered as functional relations. Atomic formulae (which we will call atomic constraints) are of the form $A(x)$ or $x f y$, where $x, y$ are first order variables, $A$ is some sort predicate and $f$ is a feature (written in infix notation). Then we can express the above feature description by the (admittedly less suggestive) formula

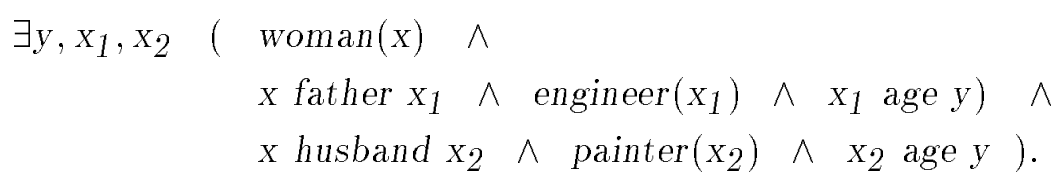

This feature logic has been investigated in detail. A complete axiomatization of the standard model (so-called feature graphs) is given in [BS93]. There it was shown that the standard model is elementarily equivalent to a tree model. Additionally, a connection to first order constructor terms has been examined [ST92].

In this paper we will be concerned with an extension to feature descriptions introduced as "functional uncertainty" by Kaplan and Zaenen [KZ88], and Kaplan and Maxwell [KM88]. This extension is made by adding a subterm 
relation, where the allowed paths for the subterms are restricted to any given regular language. It was invented for handling so-called long-distance dependencies in the grammar formalism LFG [KB82]. For a detailed description the reader is referred to [KZ88]. Further applications can be found in [Kel91].

To accomplish this extension we must first generalize the constraints of the form $x f y$ to constraints of the form $x w y$, where $w=f_{1} \ldots f_{n}$ is a string of features (called a feature path). Such feature paths are interpreted using simple relational composition.

This generalization is just syntactic sugar (see Smolka [Smo88]). This is no longer the case if we add functional uncertainty in form of constraints $x L y$, where $L$ is a regular expression denoting a regular language of feature paths. A constraint $x L y$ holds if there is a word $w \in L$ such that $x w y$ holds. By this existential interpretation a constraint $x L y$ can be seen as the disjunction

$$
x L y=\bigvee\{x w y \mid w \in L\} .
$$

As this disjunction may be infinite, functional uncertainty yields additional expressivity. Note that the constraint $x w y$ is a special case of a functional uncertainty constraint.

Kaplan and Maxwell [KM88] have shown that the satisfiability problem of the pure existential fragment (i.e. the satisfiability of formulae built with $A(x), x L y$ and equations $x \doteq y)$ is decidable, provided that a certain acyclicity condition is met. Baader et al. [BBN $\left.{ }^{+} 91\right]$ have shown that satisfiability is undecidable if we add unrestricted negation. It has, however, remained an open problem whether satisfiability of the purely existential fragment is decidable in the absence of additional conditions (such as acyclicity). In this paper we will show that it is indeed decidable.

\section{The Method}

We will first sketch the method for testing satisfiability of the standard feature descriptions, and then turn to the systems as extended by functional uncertainty. To get a good intuition note that some sort of tree model is canonical for satisfiability; a pure existential formula is satisfiable if it is satisfiable in this tree model. Thus, the feature paths used in the language can be compared directly with paths in trees.

Consider a clause $\phi=x p_{1} y_{1} \wedge x p_{2} y_{2}$ (in the rest of the paper we will call pure conjunctive formulae clauses). Although only subterm relations for $x, y_{1}$ and $x, y_{2}$ are contained in this clause, an additional subterm or equality relation 
can be implied depending on the paths $p_{1}$ and $p_{2}$. If $p_{1}$ equals $p_{2}$, we know that $y_{1}$ and $y_{2}$ must be equal, which implies that $\phi$ is equivalent to $x p_{1} y_{1} \wedge y_{1} \doteq y_{2}$. If $p_{1}$ is a prefix of $p_{2}$ and hence $p_{2}=p_{1} p^{\prime}$, we can transform $\phi$ into the equivalent formula $x p_{1} y_{1} \wedge y_{1} p^{\prime} y_{2}$, thus additionally stating that $y_{2}$ is a subterm of $y_{1}$. The reverse case is handled similarly. If neither prefix nor equality holds between the paths, there is nothing to do. By and large, clauses where the last condition holds for every $x$ and every pair of different constraints $x p_{1} y_{1} \in \phi$ and $x p_{2} y_{2} \in \phi$ are the solved forms of [Smo88], which are satisfiable.

If we consider a clause of the form $\phi=x L_{1} y_{1} \wedge x L_{2} y_{2}$, then we have again to check the relation between $y_{1}$ and $y_{2}$. But now there is in general no unique relation determined by $\phi$, since this depends on which paths $p_{1}$ and $p_{2}$ are used out of $L_{1}$ and $L_{2}$. Hence, we have to select non-deterministically a relation between $p_{1}$ and $p_{2}$ before we can calculate the relation between $y_{1}$ and $y_{2}$. In the following, we will often just say "guess" instead of "select non-deterministically".

But there is a problem with the original syntax, namely that it does not allow one to express any relation between the chosen paths ${ }^{1}$. Therefore, we extend the syntax by introducing so-called path variables (written $\alpha, \beta, \alpha^{\prime}, \ldots$ ), which are interpreted as feature paths. If we use in addition the modified subterm relation $x \alpha y$ and a restriction constraint $\alpha \dot{\epsilon} L$, a path expression $x L y$ can be expressed by the equivalent clause $x \alpha y \wedge \alpha \dot{\epsilon} L$ ( $\alpha$ new).

Using this extended (two-sorted) syntax we are now able to reason about the relations between different path variables. To do this we introduce additional constraints $\alpha \doteq \beta$ (equality), $\alpha \dot{\prec} \beta$ (prefix) and $\alpha \dot{I I} \beta$ (divergence). Divergence holds if neither equality nor prefix does. Now we can describe a normal form equivalent to the solved clauses in Smolka's work, which we will call pre-solved clauses. A clause $\phi$ is pre-solved if for each pair of different constraints $x \alpha y_{1}$ and $x \beta y_{2}$ in $\phi$ there is a constraint $\alpha$ II $\beta$ in $\phi$. Additionally, we require pre-solved clauses to contain at most one constraint $\alpha \dot{\in} L$ for each path variable $\alpha$. We call these clauses pre-solved, since these clauses are not necessarily satisfiable: it may happen that the divergence constraints together with the restrictions of the form $\alpha \dot{\epsilon} L$ are inconsistent (think of the clause $\alpha \dot{\in} f^{+} \wedge \beta \dot{\epsilon} f f^{+} \wedge \alpha \dot{\mathrm{I}} \beta$, e.g.). But pre-solved clauses have the property that if we find a valuation for the path variables, then the clause is satisfiable.

Our algorithm first transforms a clause into a set of pre-solved clauses, which

\footnotetext{
${ }^{1}$ Maxwell and Kaplan solved this problem by using operations on regular languages such as intersection and calculating prefix languages directly. The use of this method forced them to introduce a new variable each time a transformation rule was applied. For a feature description that contains a cycle of the form $x L_{1} y_{1} \wedge \ldots y_{n-1} L_{n} x$ this resulted in the introduction of an infinite number of variables.
} 
is (when viewed as a disjunction) equivalent to the initial clause. In a second phase the pre-solved clauses are checked for satisfiability with respect to the path variables. In both phases we use a set of deterministic and nondeterministic transformation rules.

Before starting with the technical part we will illustrate the first phase, since it is the more difficult. For the rest of the paper we will write clauses as sets of atomic constraints. Consider the clause $\gamma=\left\{x \alpha y, \alpha_{1} \dot{\in} L_{1}, x \beta z, \beta \dot{\epsilon} L_{2}\right\}$. Initially, one guesses the relation between the path variables $\alpha$ and $\beta$. In our example there are four different possibilities. Therefore, $\gamma$ can be expressed equivalently by the set of clauses

$$
\begin{aligned}
& \gamma_{1}=\left\{\alpha \dot{\amalg} \beta, x \alpha y, \alpha \dot{\epsilon} L_{1}, x \beta z, \beta \dot{\epsilon} L_{2}\right\} \\
& \gamma_{2}=\left\{\alpha \dot{=} \beta, x \alpha y, \alpha \dot{\epsilon} L_{1}, x \beta z, \beta \dot{\epsilon} L_{2}\right\} \\
& \gamma_{3}=\left\{\alpha \dot{\prec} \beta, x \alpha y, \alpha \dot{\epsilon} L_{1}, x \beta z, \beta \dot{\epsilon} L_{2}\right\} \\
& \gamma_{4}=\left\{\beta \dot{\imath} \alpha, x \alpha y, \alpha \dot{\epsilon} L_{1}, x \beta z, \beta \dot{\epsilon} L_{2}\right\} .
\end{aligned}
$$

The clause $\gamma_{1}$ is pre-solved. For the others we must evaluate the relation between $\alpha$ and $\beta$ as follows. In $\gamma_{2}$ we substitute $\alpha$ for $\beta$ and $y$ for $z$, which yields

$$
\left\{y \doteq z, x \alpha y, \alpha \dot{\in} L_{1}, \alpha \dot{\epsilon} L_{2}\right\} .
$$

We keep only the equality constraint for the first order variables since we are interested only in their valuation. Combining $\left\{\alpha \dot{\epsilon} L_{1}, \alpha \dot{\epsilon} L_{2}\right\}$ into $\left\{\alpha \dot{\epsilon}\left(L_{1} \cap L_{2}\right)\right\}$ will then give us an equivalent pre-solved clause. For $\gamma_{3}$ we know that the variable $\beta$ can be split up into two parts, one of them covered by $\alpha$. We can use concatenation of path variables to express this, that means we can replace $\beta$ by the term $\alpha \cdot \beta^{\prime}$ with $\beta^{\prime}$ new. This would lead to the clause

$$
\left\{\alpha \dot{\prec} \alpha \cdot \beta^{\prime}, x \alpha y, \alpha \dot{\epsilon} L_{1}, x \alpha \cdot \beta^{\prime} z, \alpha \cdot \beta^{\prime} \dot{\epsilon} L_{2}\right\} .
$$

But this could easily be expressed more simply. First, the constraint $\alpha \dot{\gamma}$ $\alpha \cdot \beta^{\prime}$ is superfluous. Second, the constraint $x \alpha \cdot \beta^{\prime} z$ in combination with $x \alpha y$ can also be expressed by $\left\{x \alpha y, y \beta^{\prime} z\right\}$. We now obtain the clause

$$
\gamma_{3}^{\prime}=\left\{x \alpha y, \alpha \dot{\epsilon} L_{1}, y \beta^{\prime} z, \alpha \cdot \beta^{\prime} \dot{\epsilon} L_{2}\right\} .
$$

This shows that we do not need concatenation of path variables within subterm agreements, and we will avoid them for simplicity.

The only thing that remains in order to achieve a pre-solved clause is to resolve the constraint $\alpha \cdot \beta^{\prime} \dot{\in} L_{2}$. To do this we have to guess a decomposition $P, S$ of $L_{2}$ with $P \cdot S=\{p s \mid p \in P, s \in S\} \subseteq L_{2}$ such that $\alpha \dot{\epsilon} P$ and $\beta^{\prime} \dot{\epsilon} S$ holds. In general, there can be an infinite number of decompositions (think of the possible decompositions of the language $f^{*} g$ ). But as we use regular 
languages, there is a finite set of regular decompositions which covers all possibilities. Finally, reducing $\left\{\alpha \dot{\epsilon} L_{1}, \alpha \dot{\epsilon} P\right\}$ to $\left\{\alpha \dot{\epsilon}\left(L_{1} \cap P\right)\right\}$ will yield a pre-solved clause.

Note that the evaluation of the prefix relation in $\gamma_{3}$ has the additional effect of introducing a new constraint $y \beta^{\prime} z$. In general this implies that after the evaluation of prefix constraints there may be some path variables whose relation is unknown. Hence, after reducing the terms of form $\alpha \doteq \beta$ or $\alpha \dot{\prec} \beta$, we may have to repeat the non-deterministic choice of relation between path variables. In the end, the only remaining constraints between path variables are of form $\alpha \dot{I I} \beta$.

Now let's turn to an additional point we have to consider, namely that the rules we present will (naturally) loop in some cases. Roughly speaking, one can say that this occurs if a cycle in the graph co-incides with a cycle in the regular language. To see this let us vary the above example and let $\gamma$ be the clause

$$
\left\{x \alpha x, \alpha \dot{\epsilon} f, x \beta z, \beta \dot{\epsilon} f^{*} g\right\}
$$

Then a possibly looping derivation could be

$$
\begin{array}{lc}
\left\{\alpha \dot{\prec} \beta, x \alpha x, \alpha \dot{\epsilon} f, x \beta z, \beta \dot{\epsilon} f^{*} g\right\} & \text { adding relation } \alpha \dot{\prec} \beta \\
\left\{x \alpha x, \alpha \dot{\epsilon} f, x \beta^{\prime} z, \alpha \cdot \beta^{\prime} \dot{\in} f^{*} g\right\} & \text { splitting } \beta \text { into } \alpha \cdot \beta^{\prime} \\
\left\{x \alpha x, \alpha \dot{\epsilon} f, x \beta^{\prime} z, \alpha \dot{\epsilon} f^{*}, \beta^{\prime} \dot{\epsilon} f^{*} g\right\} & \text { decomposing } \alpha \cdot \beta^{\prime} \dot{\in} f^{*} g \\
\left\{x \alpha x, \alpha \dot{\epsilon} f, x \beta z, \beta^{\prime} \dot{\in} f^{*} g\right\} & \text { joining } \alpha \text {-restrictions }
\end{array}
$$

But we will prove that we get a quasi-terminating rule system, which means that the rule system may cycle, but produces only finitely many different clauses (see [Der87]). This is achieved by the following measures: first, we will guarantee that the rules do not introduce additional variables; second, we restrict concatenation to length 2 ; and third, we will show that the rule system produces only finitely many regular languages. In order to show that our rewrite system is complete, we must additionally show that every solution can be found in a pre-solved clause.

\section{Preliminaries}

Throughout this paper we assume a signature consisting of a set of sorts $\mathcal{S}(A, B, \ldots)$, features $\mathcal{F}(f, g \ldots)$, first order variables $\mathcal{X}(x, y, \ldots)$ and path variables $\mathcal{P}(\alpha, \beta, \ldots)$. We use a finite set of features and infinite sets of variables and sorts. The sets $\mathcal{S}, \mathcal{F}, \mathcal{X}$ and $\mathcal{P}$ are pairwise disjoint. 
A path is a finite string of features. We say that a path $u$ is a prefix of a path $v$ (written $u \prec v$ ) if there is a non-empty path $w$ such that $v=u w$. Note that $\prec$ is neither symmetric nor reflexive. We say that two paths $u, v$ diverge (written $u \mathrm{II} v$ ) if there are features $f, g$ with $f \neq g$, and possibly empty paths $w, w_{1}, w_{2}$, such that $u=w f w_{1} \wedge v=w g w_{2}$. It is clear that II is a symmetric relation.

Proposition 3.1 Given two paths $u$ and $v$, then exactly one of the relations $u=v, u \prec v, u \succ v$ or $u \amalg v$ holds.

A path term $(p, q, \ldots)$ is either a path variable $\alpha$ or a concatenation of path variables $\alpha \cdot \beta$. We will allow complex path terms only in divergence and restriction constraints, but not in prefix or equality constraints. The set of atomic constraints is given by

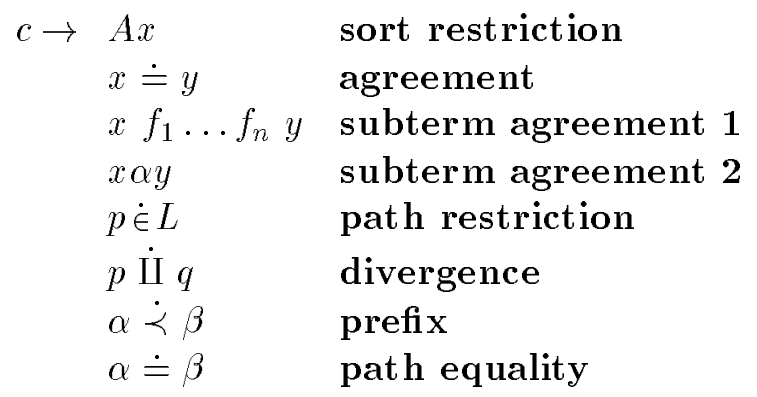

We exclude empty paths in subterm agreements, since $x \epsilon y$ is equivalent to $x \doteq y$. Therefore, we require $f_{1} \ldots f_{n} \in \mathcal{F}^{+} . L$ is a regular expression denoting a regular language $\mathcal{L}(L) \subseteq \mathcal{F}^{+}$. In the following we will not differentiate between the regular expression and the language it denotes, and we will feel free to mix both.

A clause is either the special symbol $\perp$ ("false") or a finite set of atomic constraints denoting their conjunction. We will say that a path term $\alpha \cdot \beta$ is contained (or used) in some clause $\phi$ if $\phi$ contains either a constraint $\alpha \cdot \beta \dot{\epsilon} L$ or a constraint $\alpha \cdot \beta$ II $q .^{2}$ Constraints of the form $p \dot{\epsilon} L, p$ II $q, \alpha \dot{\prec} \beta$ and $\alpha \doteq \beta$ will be called path constraints.

An interpretation $\mathcal{I}$ is a standard first order structure, where every feature $f \in \mathcal{F}$ is interpreted as a binary, functional relation $F^{\mathcal{I}}$, and where sort symbols are interpreted as disjoint, unary predicates (hence $A^{\mathcal{I}} \cap B^{\mathcal{I}}=\emptyset$ for $A \neq B)$. A valuation is a pair $\left(V_{\mathcal{X}}, V_{\mathcal{P}}\right)$, where $V_{\mathcal{X}}$ is a standard first order valuation of the variables in $X$ and $V_{\mathcal{P}}$ is a function $V_{\mathcal{P}}: \mathcal{P} \rightarrow \mathcal{F}^{+}$. We define $V_{\mathcal{P}}(\alpha \cdot \beta)$ to be $V_{\mathcal{P}}(\alpha) V_{\mathcal{P}}(\beta)$.

\footnotetext{
${ }^{2}$ We will not distinguish between $p$ İ $q$ and $q$ İ $p$.
} 
The validity of an atomic constraint in an interpretation $\mathcal{I}$ under a valuation $\left(V_{\mathcal{X}}, V_{\mathcal{P}}\right)$ is defined as follows:

$$
\begin{aligned}
\left(V_{\mathcal{X}}, V_{\mathcal{P}}\right) \models_{\mathcal{I}} A x & : \Longleftrightarrow V_{\mathcal{X}}(x) \in A^{\mathcal{I}} \\
\left(V_{\mathcal{X}}, V_{\mathcal{P}}\right) \models_{\mathcal{I}} \dot{=} y & : \Longleftrightarrow V_{\mathcal{X}}(x)=V_{\mathcal{X}}(y) \\
\left(V_{\mathcal{X}}, V_{\mathcal{P}}\right) \models_{\mathcal{I}} x f_{1} \ldots f_{n} y & : \Longleftrightarrow V_{\mathcal{X}}(x) F_{1}^{\mathcal{I}} \circ \ldots \circ F_{n}^{\mathcal{I}} V_{\mathcal{X}}(y) \\
\left(V_{\mathcal{X}}, V_{\mathcal{P}}\right) \models_{\mathcal{I}} x \alpha y & : \Longleftrightarrow\left(V_{\mathcal{X}}, V_{\mathcal{P}}\right) \models_{\mathcal{I}} x V_{\mathcal{P}}(\alpha) y \\
\left(V_{\mathcal{X}}, V_{\mathcal{P}}\right) \models_{\mathcal{I}} p \dot{\epsilon} L & : \Longleftrightarrow V_{\mathcal{P}}(p) \in L \\
\left(V_{\mathcal{X}}, V_{\mathcal{P}}\right) \models_{\mathcal{I}} p \dot{\diamond} q & : \Longleftrightarrow V_{\mathcal{P}}(p) \diamond V_{\mathcal{P}}(q) \text { for } \diamond \in\{\mathrm{II}, \prec,=\}
\end{aligned}
$$

Note that subterm agreement 2 is the only constraint where an interaction between $V_{\mathcal{X}}$ and $V_{\mathcal{P}}$ happens. The validity of sort restriction, agreement and subterm agreement 1 depend only on $V_{\mathcal{X}}$ and $\mathcal{I}$. Hence, we will sometimes omit the path valuation $V_{\mathcal{P}}$ and write $V_{\mathcal{X}} \models_{\mathcal{I}} \phi$ if $\phi$ consists only of these forms of constraint and $\phi$ is valid under $\mathcal{I}$ and $V_{\mathcal{X}}$. Similar, validity of path constraints depend only on the path valuation. We will write $V_{\mathcal{P}} \models \phi$ if $\phi$ is a clause consisting of path constraints that are valid under $V_{\mathcal{P}}$.

For checking satisfiability of clauses we will use a set of deterministic and non-deterministic transformation rules. Which set of rules is used will depend on the initial clause. Let $\phi$ be a clause and $r$ be a rule instance. We say that $r$ is applicable on $\phi$ if $\phi$ matches the definition of $r$ and the application conditions noted in the definition of $r$ are satisfied. We will write $\phi \rightarrow_{r} \gamma$ if $r$ is applicable on $\phi$ and the result of the application is $\gamma$. For a set of rules $\mathcal{R}$ we say $\phi \rightarrow_{\mathcal{R}} \gamma$ if there is an $r \in \mathcal{R}$ with $\phi \rightarrow_{r} \gamma$. $\phi$ is called $\mathcal{R}$-irreducible if no rule instance $r \in \mathcal{R}$ applies to $\phi$. We will say that a clause $\phi$ is $\mathcal{R}$-reducible if $\phi$ is not $\mathcal{R}$-irreducible. A sequence

$$
\phi_{0} \rightarrow_{r_{0}} \phi_{1} \cdots \phi_{i} \rightarrow_{r_{i}} \phi_{i+1} \cdots
$$

is called a derivation. A clause $\gamma$ is called a $(\phi, \mathcal{R})$-derivative if there is a derivation from $\phi$ to $\gamma$ that uses only rule instances of $\mathcal{R}$.

Since we have a two-sorted logic, we have to redefine the notions of soundness and preservingness. For a set $\xi \subseteq \mathcal{X}$ we define $=\xi$ to be the following relation on first order valuation:

$$
V_{\mathcal{X}}={ }_{\xi} V_{\mathcal{X}}^{\prime} \quad \text { iff } \quad \text { for all } x \in \xi \text { the equation } V_{\mathcal{X}}(x)=V_{\mathcal{X}}^{\prime}(x) \text { holds. }
$$

Similar we define $={ }_{\pi}$ with $\pi \subseteq \mathcal{P}$ for path valuations. Let $\vartheta \subseteq \mathcal{X} \cup \mathcal{P}$ be a set of variables. For a given interpretation $\mathcal{I}$ we say that a valuation $\left(V_{\mathcal{X}}, V_{\mathcal{P}}\right)$ is a $\vartheta$-solution of a clause $\phi$ in $\mathcal{I}$ if there is a valuation $\left(V_{\mathcal{X}}^{\prime}, V_{\mathcal{P}}^{\prime}\right)$ in $\mathcal{I}$ such that

$$
V_{\mathcal{X}}=\mathcal{X} \cap \vartheta_{\mathcal{X}}^{\prime}, \quad V_{\mathcal{P}}={ }_{\mathcal{P} \cap \vartheta} V_{\mathcal{P}}^{\prime} \text { and }\left(V_{\mathcal{X}}^{\prime}, V_{\mathcal{P}}^{\prime}\right) \models_{\mathcal{I}} \phi .
$$




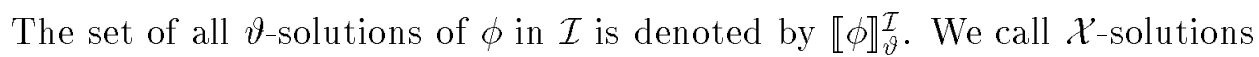
just solutions and write $\llbracket \phi \rrbracket^{\mathcal{I}}$ instead of $\llbracket \phi \rrbracket_{\mathcal{X}}^{\mathcal{I}}$. A clause $\phi$ is $\vartheta$-equivalent to a clause $\gamma$ (resp. a set of clauses $\Gamma$ ) if for every interpretation $\mathcal{I} \llbracket \phi \rrbracket^{\mathcal{I}}=\llbracket \gamma \rrbracket_{\vartheta}^{\mathcal{I}}$ (resp. $\left.\llbracket \phi \rrbracket_{\vartheta}^{\mathcal{T}}=\bigcup_{\gamma \in \Gamma} \llbracket \gamma \rrbracket_{\vartheta}^{\mathcal{T}}\right)$. Again we use equivalent as short for $V_{\mathcal{X}}$-equivalent.

A rule $R$ is $\vartheta$-sound if $\phi \rightarrow_{R} \gamma$ implies $\llbracket \phi \rrbracket_{\vartheta}^{\mathcal{T}} \supseteq \llbracket \gamma \rrbracket_{\vartheta}^{\mathcal{I}}$ for every interpretation $\mathcal{I}$. $R$ is called $\vartheta$-preserving if $\phi \rightarrow_{R} \gamma$ implies $\llbracket \phi \rrbracket_{\vartheta}^{\mathcal{I}} \subseteq \llbracket \gamma \rrbracket_{\vartheta}^{\mathcal{I}}$ for every $\mathcal{I}$. And $R$ is globally $\vartheta$-preserving if

$$
\forall \mathcal{I}: \quad \llbracket \phi \rrbracket_{\vartheta}^{\mathcal{I}} \subseteq \bigcup_{\phi \rightarrow R_{R} \gamma} \llbracket \gamma \rrbracket_{\vartheta}^{\mathcal{I}}
$$

\section{Feature Trees}

In this section we will establish two different interpretations, namely the feature tree structure and the rational feature tree structure. These interpretations are canonical for satisfiability. This means that if a clause is satisfiable, then it is also satisfiable in these interpretations. These models were introduced in [AKPS92a], [ST92] and [BS93]. In [BS93] a complete axiomatization of the the full first order theory of these models with respect to a restricted syntax has been set up. The restricted syntax uses only $A x$, $x f y$ and $x \doteq y$ as atomic constraints.

A tree domain is a nonempty set $D \subseteq \mathcal{F}^{\star}$ of paths that is prefix-closed, that is, if $w u \in D$, then $w \in D$. Note that every tree domain contains the empty path.

A feature tree is a partial function $\sigma: \mathcal{F}^{\star} \rightarrow \mathcal{S}$ whose domain is a tree domain. The paths in the domain of a feature tree represent the nodes of the tree; the empty path represents its root. We use $D_{\sigma}$ to denote the domain of a feature tree $\sigma$. A feature tree is called finite [infinite] if its domain is finite [infinite]. The letters $\sigma$ and $\tau$ will always denote feature trees.

The subtree $w^{-1} \sigma$ of a feature tree $\sigma$ at a path $w \in D_{\sigma}$ is the feature tree defined by (in relational notation)

$$
w^{-1} \sigma:=\{(q, A) \mid(w u, A) \in \sigma\} .
$$

A feature tree $\sigma$ is called a subtree of a feature tree $\tau$ if $\sigma$ is a subtree of $\tau$ at some path $w \in D_{\tau}$, and a direct subtree if $w=f$ for some feature $f$.

A feature tree $\sigma$ is called rational if (1) $\sigma$ has only finitely many distinct subtrees and (2) $\sigma$ is finitely branching (i.e., for every $w \in D_{\sigma}$, the set $\left\{w f \in D_{\sigma} \mid f \in \mathcal{F}\right\}$ is finite). Note that for every rational feature tree $\sigma$ there exist finitely many features $f_{1}, \ldots, f_{n}$ such that $D_{\sigma} \subseteq\left\{f_{1}, \ldots, f_{n}\right\}^{\star}$. 
The feature tree structure $\mathcal{T}$ is defined as follows:

- the universe of $\mathcal{T}$ is the set of all feature trees

- $\sigma \in A^{\mathcal{T}}$ iff $\sigma(\varepsilon)=A$ (i.e., $\sigma$ 's root is labeled with $A$ )

- $(\sigma, \tau) \in f^{\mathcal{T}}$ iff $f \in D_{\sigma}$ and $\tau=f^{-1} \sigma$ (i.e., $\tau$ is the subtree of $\sigma$ at $f$ ).

The rational feature tree structure $\mathcal{R}$ is the substructure of $\mathcal{T}$ consisting only of rational feature trees.

\section{$5 \quad$ Prime, Pre-Solved and Solved Clauses}

In this section, we will define the input and output clauses for both phases of the algorithm.

Let $\phi$ be some clause and $x, y$ be different variables. We say that $\phi$ binds $y$ to $x$ if $x \doteq y \in \phi$ and $y$ occurs only once in $\phi$. Here it is important that we consider equations as directed, that is, we assume that $x \doteq y$ is different from $y \doteq x$. We say that $\phi$ eliminates $y$ if $\phi$ binds $y$ to some variable $x$. A clause is called basic if it is either $\perp$ or:

1. an equation $x \doteq y$ appears in $\phi$ if and only if $\phi$ eliminates $y$; and

2. for every path variable $\alpha$ used in $\phi$ there is at most one constraint $x \alpha y \in \phi$.

A clause $\phi$ is called prime if $\phi$ is basic, $\phi$ does not contain a path term of the form $\alpha \cdot \beta$ and $\phi$ does not contain an atomic constraint of form $p$ II $q$, $\alpha \dot{\prec} \beta$ or $\alpha \doteq \beta$.

As mentioned, Kaplan and Maxwell stated the satisfiability problem for functional uncertainty in an unsorted syntax. Essentially, this syntax consists of the atomic constraints $A x, x f_{1} \ldots f_{n} y$ and $x \doteq y$ together with the additional constraint $x L y$. This constraint is interpreted as

$$
x L y=\bigvee\{x w y \mid w \in L\} .
$$

It is easy to show that every clause in this syntax can be transformed into an equivalent prime clause.

Proposition 5.1 Every clause $\phi$ in the Kaplan/Maxwell syntax can be translated into a prime clause $\gamma$ such that for every interpretation $\mathcal{I}$ and for every first order valuation $V_{\mathcal{X}}$

$$
V_{\mathcal{X}} \models_{\mathcal{I}} \phi \Longleftrightarrow \text { there is a } V_{\mathcal{P}} \text { with }\left(V_{\mathcal{X}}, V_{\mathcal{P}}\right) \models_{\mathcal{I}} \gamma \text {. }
$$


Proof. The translation can be defined by the two rewrite rules

$$
\begin{array}{ll}
\text { (Rename) } & \frac{\{x L y\} \cup \psi}{\{x \alpha y, \alpha \dot{\epsilon} L\} \cup \psi} \quad \alpha \text { new } \\
\text { (Elim) } & \frac{\{x \doteq y\} \cup \psi}{\{x \doteq y\} \cup \psi[x \leftarrow y]} \quad x \neq y, \quad x \in \operatorname{Vars}_{\mathcal{X}}(\psi)
\end{array}
$$

It is easy to check that the system consisting of these two rules will always terminate and that the result satisfies the required conditions.

This implies that it suffices to check satisfiability of prime clauses in order to check satisfiability of clauses in the Kaplan/Maxwell syntax. Hence, prime clauses are the input clauses for the first phase.

Now we turn to the output clauses of the first phase. A basic clause is said to be pre-solved if it is either $\perp$ or the following hold:

1. $A x \in \phi$ and $B x \in \phi$ implies $A=B$.

2. $\alpha \dot{\epsilon} L \in \phi$ and $\alpha \dot{\epsilon} L^{\prime} \in \phi$ implies $L=L^{\prime}$.

3. $\alpha \dot{\epsilon} \emptyset$ is not in $\phi$.

4. $\phi$ contains no terms of form $\alpha \cdot \beta$,

5. $\phi$ contains no constraints of form $\alpha \doteq \beta$ or $\alpha \dot{\prec} \beta$,

6. $\alpha$ II $\beta \in \phi$ if and only if $\alpha \neq \beta, x \alpha y \in \phi$ and $x \beta z \in \phi$.

Lemma 5.2 Let $\phi$ be a pre-solved clause different from $\perp$. Then $\phi$ is satisfiable iff there is a path valuation $V_{\mathcal{P}}$ with $V_{\mathcal{P}}=\phi_{p}$, where $\phi_{p}$ is the set of path constraints in $\phi$.

Proof. Without loss of generality we can assume that for every $x \in$ $\operatorname{Vars}_{\mathcal{X}}(\phi)$ there is a sort restriction $A x \in \phi$. Let

$$
\gamma=\left\{x V_{\mathcal{P}}(\alpha) y \mid x \alpha y \in \phi\right\} \cup\{A x \in \phi\} .
$$

Then for each interpretation $\mathcal{I}$ and each first order valuation $V_{\mathcal{X}}$ we have $\left(V_{\mathcal{X}}, V_{\mathcal{P}}\right) \models_{\mathcal{I}} \phi$ iff $V_{\mathcal{X}} \models_{\mathcal{I}} \gamma$.

Let $\mathcal{T}$ be the feature tree model as defined before. For a feature tree $\sigma$ and each word $w \in \mathcal{F}^{+}$we define $w \sigma$ to be the feature tree

$$
w \sigma=\{(w u, A) \mid(u, A) \in \sigma\} .
$$


It is easy to check that $w^{-1} w \sigma=\sigma$ holds, but not in general $w w^{-1} \sigma=\sigma$.

For every $n \in \mathcal{N}$ we define $V_{\mathcal{X}}^{n}$ to be the following first order valuation on $\operatorname{Vars} \mathcal{X}(\gamma)$ :

1. $V_{\mathcal{X}}^{0}(x)=\{(\epsilon, A)\}$, where $A x \in \gamma$,

2. $V_{\mathcal{X}}^{n+1}(x)=\{(\epsilon, A)\} \biguplus \biguplus_{x w y \in \gamma} w V_{\mathcal{X}}^{n}(y)$, where $A x \in \gamma$.

The union in the definition of $V_{\mathcal{X}}^{n+1}(x)$ is a disjoint union as $w \in \mathcal{F}^{+}$and $\forall x w y, x u z \in \gamma: w \neq u \Rightarrow w$ II $u$ by the pre-solved conditions 5-6. Thus we can prove by induction that for each $n \geq 1$

1. $x w y \in \gamma$ implies $w^{-1} V_{\mathcal{X}}^{n}(x)=V_{\mathcal{X}}^{n-1}(y)$.

2. $V_{\mathcal{X}}^{n}(x) \supseteq V_{\mathcal{X}}^{n-1}(x)$ and

3. $V_{\mathcal{X}}^{n}(x)$ is a partial function $\mathcal{F}^{\star} \rightarrow \mathcal{S}$.

Now we define $V_{\mathcal{X}}$ to be the valuation with

$$
V_{\mathcal{X}}(x)=\bigcup_{n \in \mathcal{N}} V_{\mathcal{X}}^{n}(x)
$$

By the above propositions for $V_{\mathcal{X}}^{n}$ we know that $w^{-1} V_{\mathcal{X}}(x)=V_{\mathcal{X}}(y)$ holds for each $x w y \in \gamma$. Although $V_{\mathcal{X}}(x)$ is a partial function $\mathcal{F}^{\star} \rightarrow \mathcal{S}$ for every $x$, it is not yet a valuation in $\mathcal{T}$ since the $V_{\mathcal{X}}(x)$ are not necessarily prefix-closed. This can be overcome by defining $V_{\mathcal{X}}^{\prime}$ to be the valuation

$$
\begin{aligned}
V_{\mathcal{X}}^{\prime}(x)=V_{\mathcal{X}}(x) \uplus\{(w, A) \mid & \forall C:(w, C) \notin V_{\mathcal{X}}(x) \\
& \left.\wedge \exists u \neq \epsilon, B:(w u, B) \in V_{\mathcal{X}}(x)\right\},
\end{aligned}
$$

where $A$ is an arbitrary but fixed sort symbol. Then again $w^{-1} V_{\mathcal{X}}(x)=$ $V_{\mathcal{X}}(y)$ holds for each $x w y \in \gamma$. This implies that $V_{\mathcal{X}}^{\prime}(x) w^{T^{T}} V_{\mathcal{X}}^{\prime}(y)$ holds and hence $V_{\mathcal{X}}^{\prime} \models_{\mathcal{T}} \gamma$. But then we get $\left(V_{\mathcal{X}}^{\prime}, V_{\mathcal{P}}\right) \models_{\mathcal{T}} \phi$. Since the feature trees $V_{\mathcal{X}}^{\prime}(x)$ are even rational, we get also $\left(V_{\mathcal{X}}, V_{\mathcal{P}}\right) \models_{\mathcal{R}} \phi$

Note that this implies that the structure $\mathcal{T}$ (resp. $\mathcal{R}$ ) is canonical for presolved clauses; that is, a normal form clause is satisfiable if it is satisfiable in $\mathcal{T}$ (resp. $\mathcal{R}$ ). Since in the first phase we transform each prime clause into an equivalent set of pre-solved clauses, we know that $\mathcal{T}$ is also canonical for prime clauses.

In the second phase we will check satisfiability of a pre-solved clause by transforming it into an equivalent set of solved clauses. A clause $\phi$ is called solved if it is either $\perp$ or 
1. $A x \in \phi$ and $B x \in \phi$ implies $A=B$.

2. $\alpha \dot{\epsilon} L \in \phi$ and $\alpha \dot{\epsilon} L^{\prime} \in \phi$ implies $L=L^{\prime}$.

3. $\alpha \dot{\in} \emptyset$ is not in $\phi$.

4. $\phi$ contains no terms of form $\alpha \cdot \beta$,

5. $\phi$ contains no constraints of form $\alpha \doteq \beta, \alpha \dot{\prec} \beta$ or $\alpha$ II $\beta$.

6. for every pair of variables $\alpha, \beta$ such that $\alpha \neq \beta, x \alpha y \in \phi$ and $x \beta z \in \phi$ we have $\phi \models \alpha$ İ $\beta$.

Here $\phi \models \gamma$ means that for every $\mathcal{I}$ and every $\left(V_{\mathcal{X}}, V_{\mathcal{P}}\right)$ in $\mathcal{I} \quad\left(V_{\mathcal{X}}, V_{\mathcal{P}}\right) \models_{\mathcal{I}}$ $\phi$ implies $\left(V_{\mathcal{X}}, V_{\mathcal{P}}\right) \models_{\mathcal{I}} \gamma$. Note that the definition of pre-solvedness and solvedness differ in the last two conditions and that every solved clause is also a prime clause.

Lemma 5.3 Every solved clause different from $\perp$ is satisfiable.

Proof. For every solved clause $\phi$ there is a $V_{\mathcal{X}} \cup V_{\mathcal{P}}$-equivalent clause $\gamma$ such that $\gamma$ is pre-solved. Thus, a solved clause $\phi$ is (by lemma 5.2) satisfiable if there is a path valuation $V_{\mathcal{P}}$ with $V_{\mathcal{P}} \models \phi$. But this is guaranteed by the conditions $2-5$ in the definition of solvedness.

\section{The First Phase}

\subsection{A Set of Rules}

The first rule is the non-deterministic addition of relational constraints between path variables. In one step we will add the relations between one fixed variable $\alpha$ and all other path variables $\beta$ which are used under the same node $x$ as $\alpha$. We will consider only the constraints $\alpha \doteq \beta, \alpha \dot{I} \beta$ and $\alpha \dot{\prec} \beta$ but not $\alpha \dot{\succ} \beta$. Thus the rule can be described by the following pseudo code:

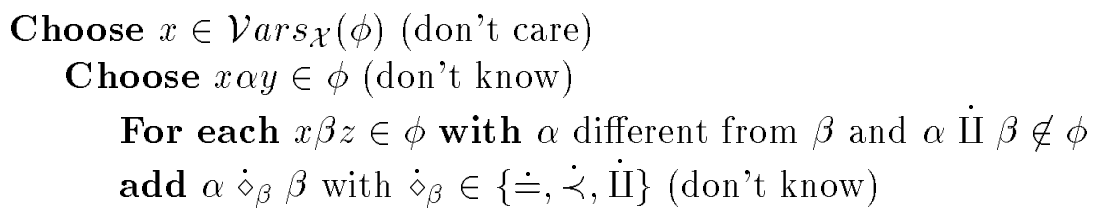

Formally, this rule is written as 
(PathRel) $\frac{\{x \alpha y\} \cup \psi}{\left\{\alpha \dot{\delta}_{\beta} \beta \mid x \beta z \in \psi \wedge \alpha \neq \beta \wedge \alpha \dot{\amalg} \beta \notin \psi\right\} \cup\{x \alpha y\} \cup \psi}$

where $\dot{\diamond}_{\beta} \in\{\dot{\doteq}, \dot{\prec}, \dot{\mathrm{II}}\}$.

This rule will only by applied if

- $\psi$ contains no prefix and path equality constraint,

- $\psi$ contains no path concatenation,

- the rule adds at least one constraint.

Although we have restricted the relations $\dot{\diamond}_{\beta}$ to $\{\dot{\doteq}, \dot{\prec}, \dot{I I}\}$, this rule is globally preserving since we have non-deterministically chosen $x \alpha y$. To see this let $\phi$ be a clause, $\mathcal{I}$ be an interpretation and $\left(V_{\mathcal{X}}, V_{\mathcal{P}}\right)$ be a valuation in $\mathcal{I}$ with $\left(V_{\mathcal{X}}, V_{\mathcal{P}}\right) \models_{\mathcal{I}} \phi$. To find an instance of (PathRel) such that $\left(V_{\mathcal{X}}, V_{\mathcal{P}}\right) \models_{\mathcal{I}} \gamma$ where $\gamma$ is the result of applying this instance, we choose $x \alpha y \in \phi$ with $V_{\mathcal{P}}(\alpha) \prec$-minimal in

$$
\left\{V_{\mathcal{P}}(\beta) \mid x \beta z \in \phi\right\} .
$$

Then for each $x \beta z \in \phi$ with $\alpha \neq \beta$ and $\alpha$ II $\beta \notin \phi$ we add $\alpha \dot{s}_{\beta} \beta$ where $V_{\mathcal{P}}(\alpha) \diamond_{\beta} V_{\mathcal{P}}(\beta)$ holds. Note that $\dot{\delta}_{\beta}$ equals $\dot{\succ}$ will not occur since we have chosen a path variable $\alpha$ the interpretation of which is $\prec$-minimal. Therefore, the restriction $\dot{\diamond}_{\beta} \in\{\dot{=}, \dot{\prec}, \dot{\mathrm{II}}\}$ is satisfied.

The definition of (PathRel) is more complex than the naive one in the introduction. The reason for this is that only by using this special definition can we maintain the condition that concatenation of path variables is restricted to binary concatenation. To see this suppose that we had added both $\beta_{1} \dot{\prec} \alpha$ and $\alpha \dot{\prec} \beta_{2}$ to a clause $\gamma$. Then first splitting up the variable $\beta_{2}$ into $\alpha \cdot \beta_{2}^{\prime}$ and then $\alpha$ into $\beta_{1} \cdot \alpha^{\prime}$ will result in a substitution of $\beta_{2}$ in $\gamma$ by $\beta_{1} \cdot \alpha^{\prime} \cdot \beta_{2}^{\prime}$. By the definition of (PathRel) we have ensured that this does not happen.

The second non-deterministic rule is used in the decomposition of regular languages. For decomposition we have the following rules:

$$
\begin{array}{lll}
\text { (DecClash) } & \frac{\{\alpha \cdot \beta \dot{\epsilon} L\} \cup \psi}{\perp} & \text { if }\{w \in L|| w \mid>1\}=\emptyset \\
\text { (LangDec } \left._{\Lambda}\right) & \frac{\{\alpha \cdot \beta \dot{\epsilon} L\} \cup \psi}{\{\alpha \dot{\epsilon} P\} \cup\{\beta \dot{\epsilon} S\} \cup \psi} & P \cdot S \subseteq L
\end{array}
$$

where $L, P, S \subseteq F^{+}$and $\Lambda$ is a given finite set of reg. languages with $L, P, S \in \Lambda . \quad L$ must contain a path $w$ with $|w|>1$. 
The clash rule is needed since we require regular languages not to contain the empty path.

We use $\Lambda$ in (LangDec $\Lambda$ ) as a global restriction, which means that for every $\Lambda$ we get a different rule ( $\operatorname{LangDec}_{\Lambda}$ ) (and hence a different rule system $\mathcal{R}_{\Lambda}$ ). This is done as the rule system is quasi-terminating. By restricting (LangDec ${ }_{\Lambda}$ ) we can guarantee that only finitely many regular languages are produced.

For ( $\operatorname{LangDec}_{\Lambda}$ ) to be globally preserving we need to find, for every possible valuation of $\alpha$ and $\beta$, a suitable pair $P, S$ in $\Lambda$. Therefore, we require $\Lambda$ to satisfy

$$
\begin{aligned}
& \forall L \in \Lambda, \forall w_{1}, w_{2} \neq \epsilon: \\
& \quad\left[w_{1} w_{2} \in L \Rightarrow \exists P, S \in \Lambda:\left(P \cdot S \subseteq L \wedge w_{1} \in P \wedge w_{2} \in S\right)\right] .
\end{aligned}
$$

We will call $\Lambda$ closed under decomposition if it satisfies this condition. Additionally, we have to ensure that $L \in \Lambda$ for every $L$ that is contained in some clause $\phi$. We will call such a set $\Lambda \phi$-closed.

The remaining rules are listed in figure 1 . Note that we have not considered clauses containing subterm agreement 1 , since these constraint are superfluous for checking satisfiability. A constraint $x f_{1} \ldots f_{n} y$ can be expressed by the equivalent clause $\left\{x \alpha y, \alpha \dot{\epsilon} f_{1} \ldots f_{n}\right\}$ ( $\alpha$ new).

The (Pre) rule needs some additional explanation. One might expect (Pre) to be of the form

$$
\left(\text { Pre' }^{\prime}\right) \frac{\{\alpha \dot{\prec} \beta\} \cup\{x \alpha y\} \cup\{x \beta z\} \cup \psi}{\{x \alpha y\} \cup\left\{y \beta^{\prime} z\right\} \cup \psi\left[\beta \leftarrow \alpha \cdot \beta^{\prime}\right]} \quad \beta^{\prime} \text { new. }
$$

But as we have mentioned, we have to define our rules in a way such that no additional variables are introduced. This is not satisfied by the rule (Pre'). For solving this problem note that $\beta$ is not used in the result of applying (Pre'). Hence, we can substitute $\beta^{\prime}$ by $\beta$, which has the effect that no new variable is needed. This leads to the definition of (Pre) as presented in figure 1.

The following proposition and lemma will show that the definition of ( LangDec $_{\Lambda}$ ) is meaningful.

Proposition 6.1 If $\Lambda$ is $\phi$-closed and closed under intersection, then $\Lambda$ is $\gamma$-closed for all $\left(\phi, \mathcal{R}_{\Lambda}\right)$-derivatives $\gamma$.

Proof. We will prove this lemma by induction over the length of derivations. We use the term $\operatorname{reg}(\gamma)$ to denote the set of regular languages used in $\gamma$. Then $\mathcal{R}_{\Lambda}$ is $\gamma$-closed if $\operatorname{reg}(\gamma) \subseteq \Lambda$. 


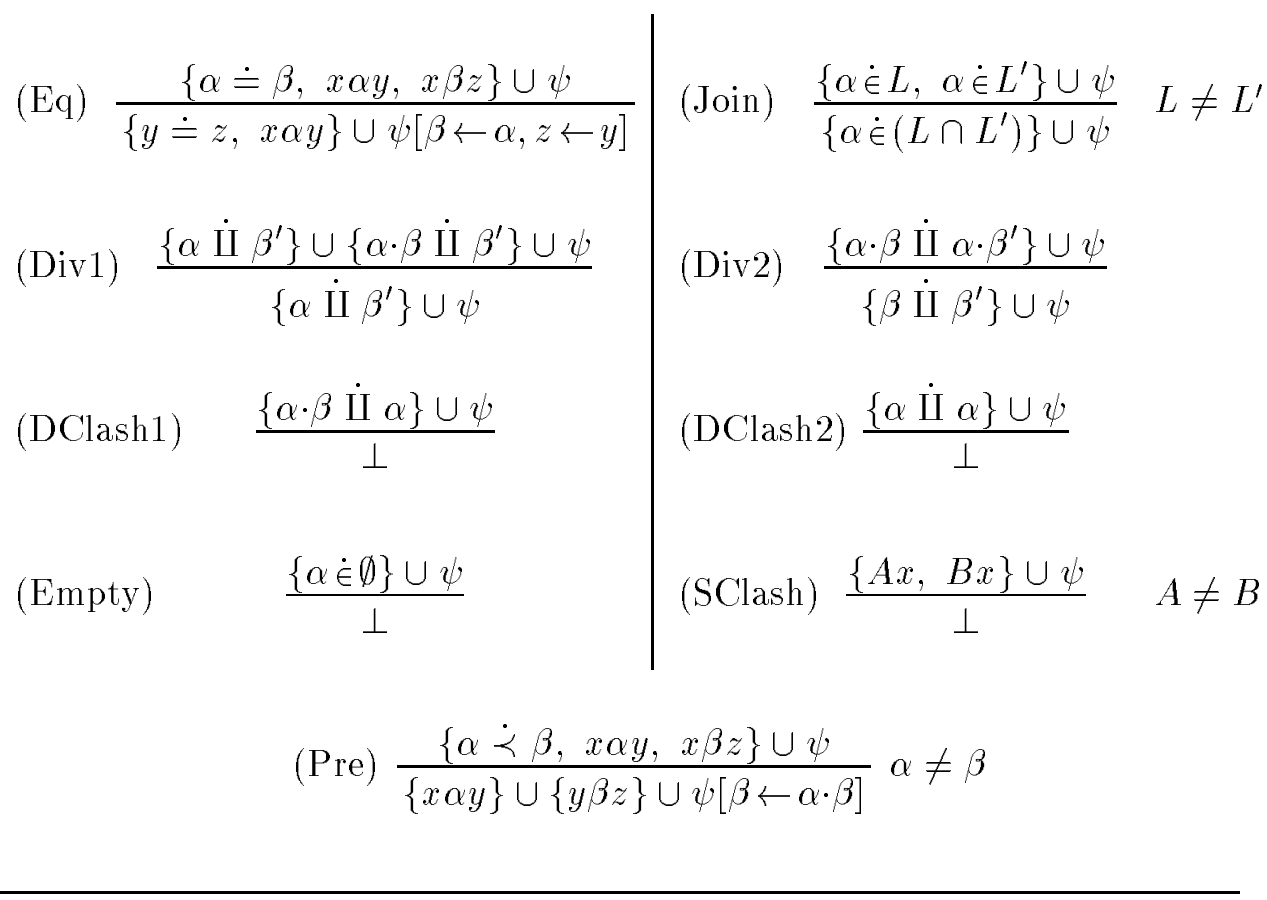

Figure 1: Simplification rules 
Let $\gamma$ be some $(\phi, \mathcal{R})$-derivative. For the base step $\gamma=\phi$ the lemma holds trivially. For the induction step let $\gamma$ satisfy the induction hypotheses $\operatorname{reg}(\gamma) \subseteq \Lambda$ and let $r \in \mathcal{R}_{\Lambda}$ be a rule such that $\gamma \rightarrow_{r} \gamma^{\prime}$.

If $r$ is some clash rule, then $\operatorname{reg}\left(\gamma^{\prime}\right)=\emptyset$.

If $r$ is not a clash rule and not in $\left(\operatorname{LangDec}_{\Lambda}\right)$ or $(\operatorname{Join})$, then $\operatorname{reg}\left(\gamma^{\prime}\right)=\operatorname{reg}(\gamma)$ and therefore $\operatorname{reg}\left(\gamma^{\prime}\right) \subseteq \Lambda$ by induction hypotheses. If $r \in\left(\operatorname{LangDec}_{\Lambda}\right)$, then $r$ adds only regular languages $P, S \in \Lambda$.

Now let

$$
r^{\prime}=\frac{\left\{\alpha \dot{\epsilon} L, \alpha \dot{\epsilon} L^{\prime}\right\} \cup \psi}{\left\{\alpha \dot{\epsilon}\left(L \cap L^{\prime}\right)\right\} \cup \psi} \in \text { (Join). }
$$

By induction hypotheses we know that $L, L^{\prime} \in \Lambda$. But then $\left(L \cap L^{\prime}\right) \in \Lambda$ since $\Lambda$ is closed under intersection.

Lemma 6.2 For every prime clause $\phi$ there is a finite $\Lambda$ such that $\Lambda$ is $\phi$-closed, closed under intersection and decomposition.

Proof. We define a deterministic automaton $\mathcal{A}$ over $\mathcal{F}$ to be a tuple $\left(Q_{\mathcal{A}}, i_{\mathcal{A}}, \delta_{\mathcal{A}}, \operatorname{Fin}_{\mathcal{A}}\right)$, where

1. $Q_{\mathcal{A}}$ is a finite set of states,

2. $i_{\mathcal{A}} \in Q_{\mathcal{A}}$ is the initial state,

3. $\delta_{\mathcal{A}}: Q_{\mathcal{A}} \times \mathcal{F} \rightarrow Q_{\mathcal{A}}$ is a transition function,

4. and $\operatorname{Fin}_{\mathcal{A}} \subseteq Q_{\mathcal{A}}$ are the final states.

With $\delta_{\mathcal{A}}^{*}$ we mean the unique extension of $\delta_{\mathcal{A}}$ to $\mathcal{F}^{*}$. The regular language that is accepted by an automaton $\mathcal{A}$ is defined as

$$
L(\mathcal{A})=\left\{w \mid \delta_{\mathcal{A}}^{*}\left(i_{\mathcal{A}}, w\right) \in \operatorname{Fin}_{\mathcal{A}}\right\} .
$$

Let $\operatorname{reg}(\phi)=\left\{L_{1}, \ldots, L_{n}\right\} \subseteq P\left(\mathcal{F}^{+}\right)$be the set of regular languages used in $\phi$ and let $\mathcal{A}_{i}=\left(Q_{\mathcal{A}_{i}}, i_{\mathcal{A}_{i}}, \delta_{\mathcal{A}_{i}}\right.$, Fin $\left._{\mathcal{A}_{i}}\right)$ be finite, deterministic automatons such that $\mathcal{A}_{i}$ accepts $L_{i}$. For each $\mathcal{A}_{i}$ we define $\operatorname{dec}\left(\mathcal{A}_{i}\right)$ to be the set

$$
\operatorname{dec}\left(\mathcal{A}_{i}\right)=\left\{\overline{L_{p}^{q}} \mid p, q \in Q_{\mathcal{A}_{i}}\right\},
$$

where $\overline{L_{p}^{q}}=\left\{w \in \mathcal{F}^{+} \mid \delta_{\mathcal{A}_{i}}^{*}(p, w)=q\right\}$.

Of course, each $\operatorname{dec}\left(\mathcal{A}_{i}\right)$ is finite and contains $L_{i}$. Furthermore, it is also closed under decomposition. The complete set of decompositions for a language $\overline{L_{p}^{q}} \in \operatorname{dec}\left(\mathcal{A}_{i}\right)$ consists of the languages

$$
P=\overline{L_{p}^{s}} \text { and } S=\overline{L_{s}^{q}} \text { for } s \in Q_{\mathcal{A}_{i}} .
$$


We define $\Lambda_{0}$ to be $\bigcup_{i=1}^{n} \operatorname{dec}\left(\mathcal{A}_{i}\right)$. $\Lambda_{0}$ contains each $L_{i} \in \operatorname{reg}(\phi)$ and is closed under decomposition. Now let

$$
\Lambda=\mathrm{fi}\left(\Lambda_{0}\right)
$$

be the least set that contains $\Lambda_{0}$ and is closed under intersection. Then $\Lambda$ is finite and $\phi$-closed, since it contains each $L_{i} \in \operatorname{reg}(\phi)$.

We will prove that $\Lambda$ is also closed under decomposition. Given some $L \in \Lambda$ and a path $w=w_{1} w_{2} \in L$, we have to find an appropriate decomposition $P, S$ in $\Lambda$. Since each $L$ in $\Lambda$ can be written as a finite intersection

$$
L=\bigcap_{k=1}^{m} L_{k}
$$

with $L_{k}$ in $\Lambda_{0}$, we know that $w=w_{1} w_{2}$ is in $L_{k}$ for $1 . . m$. As $\Lambda_{0}$ is closed under decomposition, there are languages $P_{k}$ and $S_{k}$ for $k=1 . . m$ with $w_{1} \in P_{k}, w_{2} \in S_{k}$ and $P_{k} \cdot S_{k} \subseteq L_{k}$. Let $P=\bigcap_{k=1}^{m} P_{k}$ and $S=\bigcap_{k=1}^{m} S_{k}$. Clearly, $w_{1} \in P, w_{2} \in S$ and $P \cdot S \subseteq L$. Furthermore, $P, S \in \Lambda$ as $\Lambda$ is closed under intersection. This implies that $P, S$ is an appropriate decomposition for $w_{1} w_{2}$.

\subsection{Some Properties of the Rule System}

For the rest of the paper we will call clauses that are derivable from prime clauses admissible.

\section{Lemma 6.3}

1. Every admissible clause is basic.

2. If $\alpha \dot{\prec} \beta, \alpha \doteq \beta$ or $\alpha$ II $\beta$ is contained in some admissible clause $\phi$, then there is a variable $x$ such that $x \alpha y$ and $x \beta z$ is in $\phi$.

Proof. The proof of the first claim is left to the reader. The second claim will be proved by induction over the length of derivations. For prime clauses the claim holds trivially. For the induction hypotheses assume that we have proven the claim for every admissible clause $\phi$ that is derivable from a prime clause in $n$ steps and let $\phi \rightarrow_{r} \phi^{\prime}$. If $r$ is different from (Pre), (PathRel), (Eq) or (Div2), there is nothing to prove. Thus we have the following cases:

$r \in$ (PathRel): the claim holds by definition of (PathRel). 
$r \in(\mathrm{Eq})$ : the claim is invariant under substitution of one variable $\beta$ by another variable $\alpha$ if both $x \alpha y$ and $x \beta z$ are contained in $\phi$.

$r \in$ (Pre): then $\phi=\{\alpha \dot{\prec} \beta, x \alpha y, x \beta z\} \cup \psi$ and $\phi^{\prime}=\{x \alpha y, y \beta z\} \cup \psi[\beta \leftarrow$ $\alpha \cdot \beta]$. The only subterm agreement constraint that is changed is $x \beta z$. But as $\beta$ is substituted by $\alpha \cdot \beta, \phi^{\prime}$ does not contain any path equality or prefix constraints involving $\beta$.

$r \in$ (Div2): then $\phi=\left\{\alpha \cdot \beta\right.$ İ $\left.\alpha \cdot \beta^{\prime}\right\} \cup \psi$ and $\phi^{\prime}=\left\{\beta\right.$ İ $\left.\beta^{\prime}\right\} \cup \psi$. We will prove below that if $\alpha \cdot \beta$ is contained in some admissible clause $\gamma$, then there are variables $x, y, z$ such that $x \alpha y$ and $y \beta z$ are contained in $\gamma$. This will complete the proof, since then $\alpha \cdot \beta \dot{\mathrm{II}} \alpha \cdot \beta^{\prime}$ in $\phi$ implies that there are variables $x, y, z$ and $x^{\prime}, y^{\prime}, z^{\prime}$ with $\left\{x \alpha y, y \beta z, x^{\prime} \alpha y^{\prime}, y^{\prime} \beta^{\prime} z^{\prime}\right\} \subseteq \phi$. But as $\phi$ is admissible, it is also basic by the first claim. Hence, $x$ equals $x^{\prime}$ and $y$ equals $y^{\prime}$. Therefore, both $y \beta z$ and $y \beta^{\prime} z^{\prime}$ are in $\phi$ and in $\phi^{\prime}$.

Thus it remains to show that if $\alpha \cdot \beta$ is used in some admissible clause $\gamma$, then there are variables $x, y, z$ such that $x \alpha y$ and $y \beta z$ are in $\gamma$. Let $\phi$ be an admissible clause for which this holds, and let $\gamma \rightarrow_{r} \gamma^{\prime}$. The only rules we have to consider are $(\mathrm{Eq})$ and $($ Pre). For $(\mathrm{Eq})$ note that the claim is invariant under consistent variable renaming. If $r \in$ (Pre), then we have to check the path term $\alpha \cdot \beta$ that is introduced by $r$. But by definition of (Pre) the clause $\gamma^{\prime}$ must contain both $x \alpha y$ and $y \beta z$.

This lemma implies that (Eq) can always be applied if a constraint $\alpha \doteq \beta$ is contained in some admissible clause. The next lemma will show that different applications of $(\mathrm{Pre})$ or $(\mathrm{Eq})$ will not interact. This means the application of one of these rules to some prefix or path equality constraint will not change any other prefix or path equality constraint contained in the same clause.

Lemma 6.4 Given some prime clause $\phi$ and a derivation

$$
\phi=\phi_{0} \rightarrow_{r_{0}} \phi_{1} \cdots \phi_{n-1} \rightarrow_{r_{n-1}} \phi_{n}=\gamma
$$

that contains an application of (PathRel). Then $\alpha \doteq \beta \in \gamma$ (resp. $\alpha \dot{\prec} \beta \epsilon$ $\gamma$ ) implies $\alpha \doteq \beta \in \phi_{i}$ (resp. $\alpha \dot{\prec} \beta \in \phi_{i}$ ) for $i>k$, where $k$ is the number of the last application of (PathRel). Furthermore, if $\alpha \cdot \beta$ is contained in $\gamma$, then either $\alpha \cdot \beta$ or $\alpha \dot{\prec} \beta$ is contained in $\phi_{i}$ for $i>k$.

Proof. We will use induction over length of derivations. Assume that we have proven the lemma for admissible clauses $\gamma$ that are derivable in $n$ steps 
and let $\gamma \rightarrow_{r} \gamma^{\prime}$ with $r \notin$ (PathRel). If $r$ is different from (Eq) or (Pre), then there is nothing to prove. If $r \in(\mathrm{Eq})$, then a constraint $\alpha \dot{\prec} \beta$ or $\alpha \doteq \beta$ in $\gamma^{\prime}$ can be missing in $\gamma$ if and only if $\gamma$ contains a constraint $\alpha \doteq \beta^{\prime}$ or $\alpha \dot{\prec} \beta^{\prime}$ (resp. $\beta^{\prime} \doteq \beta$ or $\beta^{\prime} \prec \beta$ ) and $r$ is of the form

$$
\frac{\left\{\beta \doteq \beta^{\prime}, \ldots\right\} \cup \psi}{\ldots} \text { with } \beta^{\prime} \neq \beta \quad\left(\text { resp. } \frac{\left\{\alpha \doteq \beta^{\prime}, \ldots\right\} \cup \psi}{\ldots} \text { with } \beta^{\prime} \neq \alpha\right) .
$$

Hence, $\gamma$ must contain at least two prefix or path equality constraints, the left sides of which are different. By induction hypotheses these path equality or prefix constraints must have been introduced by the last application of (PathRel). But this contradicts to the definition of (PathRel). A similar argument can be given for the part of the lemma concerning path terms of form $\alpha \cdot \beta$.

If $r$ is in (Pre), then we have to check only the second claim of the lemma, namely that $\alpha \cdot \beta$ contained in $\gamma^{\prime}$ implies that either $\alpha \dot{\prec} \beta$ is in $\gamma$ or $\alpha \cdot \beta$ is used in $\gamma$. For the all path terms in $\gamma^{\prime}$ that are not introduced by this application of (Pre) this holds trivially. For the path term $\alpha \cdot \beta$ that is introduced, this is guaranteed by the application condition of (Pre), namely that $\gamma$ must contain $\alpha \dot{\prec} \beta$.

We can derive from this lemma certain syntactic properties of admissible clauses which are needed for proving completeness and quasi-termination.

Corollary 6.5 If $\alpha \dot{\prec} \beta$ is contained in an admissible clause $\phi$, then $\alpha$ is different from $\beta$. Furthermore, there is no other prefix or equality constraint in $\phi$ involving $\beta$ and neither $\beta \cdot \beta^{\prime}$ nor $\beta^{\prime} \cdot \beta$ is in $\phi$.

Note that by lemma 6.3 together with this corollary, the rule (Pre) is always applicable if a constraint $\alpha \dot{\prec} \beta$ is contained in an admissible clause. Furthermore, an application of (Pre) causes no violation of the restrictions that we have imposed on the syntax. This means that concatenation does not occur in prefix or path equality constraints; and concatenation of path variables is restricted to binary concatenation.

Lemma 6.6 If $\alpha \cdot \beta$ II $\beta^{\prime}$ is contained in an admissible clause $\phi$ with $\alpha$ different from $\beta^{\prime}$, then $\phi$ contains a constraint of form $\alpha$ II $\beta^{\prime}, \alpha \doteq \beta^{\prime}$ or $\alpha \dot{\prec} \beta^{\prime}$.

Proof. We will prove a stronger result, namely that if $\left\{\alpha \dot{\prec} \beta, \beta\right.$ II $\left.\beta^{\prime}\right\} \subseteq \phi$ or $\left\{\alpha \cdot \beta \dot{I I} \beta^{\prime}\right\} \subseteq \phi$, then $\phi$ contains a constraint of form $\alpha \dot{\mathrm{II}} \beta^{\prime}, \alpha \doteq \beta^{\prime}$ or 
$\alpha \dot{\prec} \beta^{\prime}$. We will prove this by induction over length of derivations. Assume that we have proven the claim for every admissible clause $\phi$ that is derivable in $n$ steps from a prime clause and let $\phi \rightarrow_{r} \phi^{\prime}$. Again we have to check only the rules (Pre), (PathRel), (Eq) or (Div2):

$r \in\left(\right.$ PathRel): we have to check only constraints $\beta$ II $\beta^{\prime}$ that are already in $\phi$. By lemma 6.3 we know that if $\beta$ II $\beta^{\prime}$ is in $\phi$, then there is a variable $x$ with both $x \beta y$ and $x \boldsymbol{\beta}^{\prime} z$ in $\phi$. Hence, if (PathRel) adds the constraint $\alpha \dot{\prec} \beta$, it must by definition also add a constraint $\alpha \dot{\mathrm{II}} \beta^{\prime}$, $\alpha \doteq \beta^{\prime}$ or $\alpha \dot{\prec} \beta^{\prime}$.

$r \in(\mathrm{Eq})$ : the claim is invariant under consistent variable renaming.

$r \in\left(\right.$ Pre): then $\phi=\{\alpha \dot{\prec} \beta, x \alpha y, x \beta z\} \cup \psi$ and $\phi^{\prime}=\{x \alpha y, y \beta z\} \cup \psi[\beta \leftarrow$ $\alpha \cdot \beta]$. The only case that we have to check is that $\phi$ contains a constraint $\beta$ II $\beta^{\prime}$. Then $\phi^{\prime}$ contains $\alpha \cdot \beta$ II $\beta^{\prime}$. By induction hypotheses $\phi$ must contain a constraint $c$ of form $\alpha$ II $\beta^{\prime}, \alpha \doteq \beta^{\prime}$ or $\alpha \dot{\prec} \beta^{\prime}$. Since (Pre) does not change $c$, this must holds also for $\phi^{\prime}$.

$r \in$ (Div2): then $\phi=\left\{\alpha \cdot \beta\right.$ İ $\left.\alpha \cdot \beta^{\prime}\right\} \cup \psi$ and $\phi^{\prime}=\left\{\beta\right.$ İ $\left.\beta^{\prime}\right\} \cup \psi$. The only new divergence constraint that comes in is $\beta$ II $\beta^{\prime}$. But as $\phi$ contains both $\alpha \cdot \beta$ and $\alpha \cdot \beta^{\prime}$, it may not contain $\alpha \dot{\prec} \beta$ or $\alpha \dot{\prec} \beta^{\prime}$ by corollary 6.5 . Hence, $\phi^{\prime}$ does not contain such a constraint.

This lemma ensures that a constraint $\alpha \cdot \beta$ II $\beta^{\prime}$ is always reducible. If $\beta^{\prime}$ equals $\alpha$, then we could apply (DClash1). If $\alpha \dot{\text { II }} \beta^{\prime}$ is in $\phi$, we can apply (Div1). If $\alpha \doteq \beta^{\prime}$ is in $\phi$ we can apply (Eq) followed by (DClash1). If $\phi=$ $\left\{\alpha \dot{\prec} \beta^{\prime}, \alpha \cdot \beta\right.$ II $\left.\beta^{\prime}\right\} \cup \psi$, then we can apply (Pre) yielding $\left\{\alpha \cdot \beta\right.$ II $\left.\alpha \cdot \beta^{\prime}\right\} \cup \psi^{\prime}$, where we can apply (Div2).

\subsection{Soundness and Completeness}

Proposition 6.7 The rules (Eq), (Div1,2), (SClash), (Join), (Empty) and (DClash1,2) are $\mathcal{X} \cup \mathcal{P}$-sound and $\mathcal{X} \cup \mathcal{P}$-preserving.

Proposition 6.8 The rule (Pre) is $\mathcal{X}$-sound and $\mathcal{X}$-preserving.

For (Pre) we can even characterize pairs of path valuations which preserve the $\mathcal{X}$-solutions. 
Proposition 6.9 Let $\phi=\{\alpha \dot{\prec} \beta, x \alpha y, x \beta z\} \cup \psi$ and $\gamma$ be the result of applying (Pre) to $\phi$. Given a pair of path valuations $V_{\mathcal{P}}, V_{\mathcal{P}}^{\prime}$ with

$$
V_{\mathcal{P}}=_{\mathcal{P}-\{\beta\}} V_{\mathcal{P}}^{\prime} \quad \text { and } \quad V_{\mathcal{P}}(\beta)=V_{\mathcal{P}}(\alpha) V_{\mathcal{P}}^{\prime}(\beta)=V_{\mathcal{P}}^{\prime}(\alpha) V_{\mathcal{P}}^{\prime}(\beta),
$$

then for each interpretation $\mathcal{I}$ and for each first order valuation $V_{\mathcal{X}}$

$$
\left(V_{\mathcal{X}}, V_{\mathcal{P}}\right) \models_{\mathcal{I}} \phi \Longleftrightarrow\left(V_{\mathcal{X}}, V_{\mathcal{P}}^{\prime}\right) \models_{\mathcal{I}} \gamma .
$$

Proposition 6.10 If $\Lambda$ is closed under decomposition, then (LangDec ${ }_{\Lambda}$ ) is $\mathcal{X} \cup \mathcal{P}$-sound and globally $\mathcal{X} \cup \mathcal{P}$-preserving. Furthermore, (PathRel) is $\mathcal{X} \cup \mathcal{P}$-sound and globally $\mathcal{X} \cup \mathcal{P}$-preserving.

Finally, we have to prove that the rules are complete. This means that given an input clause $\phi$, for every solution $V_{\mathcal{X}}$ of $\phi$ in some interpretation $\mathcal{I}$ there is a pre-solved clause $\gamma$ derivable from $\phi$ such that $V_{\mathcal{X}}$ is a solution of $\gamma$. If the rule system is terminating, then for completeness one has to prove that the pre-solved clauses are just the irreducible clauses.

In our case this is not enough since the rule system can loop. Therefore, we have to prove explicitly that each solution of a given prime clause $\phi$ can be found in some pre-solved $\phi$-derivative. We define $\operatorname{Irred}\left(\phi, \mathcal{R}_{\Lambda}\right)$ to be the set all $\left(\phi, \mathcal{R}_{\Lambda}\right)$-derivatives which are $\mathcal{R}_{\Lambda}$-irreducible, and Pre-Solved $\left(\phi, \mathcal{R}_{\Lambda}\right)$ to be the set of all pre-solved clauses which are derivable from $\phi$. A set of rules $\mathcal{R}_{\Lambda}$ is said to be $\phi$-complete w.r.t. to a set of variables $\vartheta$ if

1. $\operatorname{Irred}\left(\phi, R_{\Lambda}\right)=\operatorname{Pre-Solved}\left(\phi, R_{\Lambda}\right)$,

2. for every interpretation $\mathcal{I}$

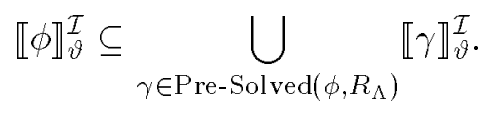

We will show that for every prime clause $\phi$ there is a set of regular languages $\Lambda$ such that $\mathcal{R}_{\Lambda}$ is $\phi$-complete w.r.t the first order variables $\mathcal{X}$.

Theorem 6.11 (Completeness I) Given a prime clause $\phi$. If $\Lambda$ is a set of regular languages that is $\phi$-closed, closed under intersection and closed under decomposition, then every $\left(\phi, \mathcal{R}_{\Lambda}\right)$-derivative $\gamma$ that is not pre-solved is $\mathcal{R}_{\Lambda}$-reducible.

Proof. Let $\gamma$ be a $\left(\phi, \mathcal{R}_{\Lambda}\right)$-derivative that is not pre-solved. We will check all conditions that are stated in the definition on page 12 .

If one of the conditions $1-3$ is not satisfied by $\gamma$, then one of the rules (SClash), (Join) or (Empty) will apply.

Now let's check the conditions 4 and 5 : 
$\gamma$ contains a constraint $\alpha \cdot \beta \dot{\epsilon} L$. As $\Lambda$ is $\phi$-closed, we know that $\Lambda$ is also $\gamma$-closed by lemma 6.1. Therefore we can apply $\left(\operatorname{LangDec}_{\Lambda}\right)$ or (DecClash).

$\gamma$ contains a constraint $\alpha \cdot \beta$ II $\alpha^{\prime} \cdot \beta^{\prime}$. By lemma 6.4 we know that $\alpha$ equals $\alpha^{\prime}$. Hence, we can apply (Div2).

$\gamma$ contains a constraint $\alpha \cdot \beta$ II $\beta^{\prime}$. If $\beta^{\prime}$ equals $\alpha$, then we can directly apply (DClash 1 ). Otherwise, there is by lemma 6.6 a constraint $\alpha \doteq$ $\beta^{\prime}, \alpha \dot{\prec} \beta^{\prime}$ or $\alpha$ II $\beta^{\prime}$ in $\gamma$. If $\alpha \doteq \beta^{\prime}$ is in $\gamma$, we can apply (Eq) by lemma 6.3. This will result in the substitution of $\beta^{\prime}$ by $\alpha$. The remaining constraint $\alpha \cdot \beta$ II $\alpha$ can be reduced using (DClash1). If $\alpha \dot{\prec} \beta^{\prime}$ is in $\gamma$, then we can apply (Pre) by lemma 6.3 and corollary 6.5 . We will obtain the constraint $\alpha \cdot \beta$ II $\alpha \cdot \beta^{\prime}$, which can be reduced using (Div2). The last case is that $\alpha$ II $\beta^{\prime}$ is in $\gamma$, where we can apply (Div1).

$\gamma$ contains a constraint $\alpha \doteq \beta$. Then $(\mathrm{Eq})$ is applicable by lemma 6.3 .

$\gamma$ contains a constraint $\alpha \prec \beta$. Then (Pre) is applicable by lemma 6.3 and corollary 6.5.

The remaining case is that $\gamma$ does not satisfy the last condition of a presolved clause, namely that $\alpha$ II $\beta$ with $\alpha \neq \beta$ in $\gamma$ if and only if $x \alpha y$ and $x \beta y$ in $\gamma$. Given the above, we can now assume that $\gamma$ does not contain a path concatenation or a prefix or path equality constraint.

There are three possibilities for $\gamma$ to violate the last condition. The first is that $\gamma$ contains a constraint of the form $\alpha \dot{I} \alpha$. Then (DClash2) is applicable. The second is that there is a constraint $\alpha \dot{\mathrm{II}} \beta$ with $x \alpha y \in \gamma$ and $x^{\prime} \beta y^{\prime} \in \gamma$ such that $x$ is different from $x^{\prime}$. But this is excluded by lemma 6.3 .

The last case is that there are different path variables $\alpha$ and $\beta$ such that $x \alpha y$ and $x \beta z$ are in $\gamma$ but $\alpha \dot{\text { II }} \beta$ is not. As $\gamma$ contains no concatenation and no path equality or prefix constraints, the rule (PathRel) is applicable.

Next we have to prove the second property for $\phi$-completeness, namely that for every interpretation $\mathcal{I}$ and for every solution $V_{\mathcal{X}}$ of $\phi$ there is a presolved $\phi$-derivative $\gamma$ with $V_{\mathcal{X}} \in \llbracket \gamma \rrbracket^{\mathcal{I}}$. This property is needed since our rule system can loop. Let us recall an example of a looping derivation in order to explain the main idea involved in the second part of the completeness proof. In contrast to our first example of a looping derivation (see page 7), we will now omit the path restrictions, since they are not needed for what we want to demonstrate. Let $\phi$ be the clause

$$
\phi=\{x \alpha x, x \beta y\} .
$$


A looping derivation can consist of an application of (PathRel) yielding the clause $\phi_{1}=\{\alpha \dot{\prec} \beta, x \alpha x, x \beta y\}$, followed by an application of (Pre) on $\gamma$ yielding $\phi_{2}=\phi{ }^{3}$ As one can imagine, the reason for looping derivation is the rule (Pre). We will later prove that indeed every infinite derivation must use the (Pre) rule infinitely often.

For proving the second completeness property we restrict the set of allowed derivations depending on some arbitrary but fixed valuation $\left(V_{\mathcal{X}}, V_{\mathcal{P}}\right)$ with $\left(V_{\mathcal{X}}, V_{\mathcal{P}}\right) \models_{\mathcal{I}} \phi$. This control will guarantee that

1. $V_{\mathcal{X}}$ is a solution of every clause in the derivation,

2. under this control, all derivations are finite.

Will we additionally show that even under this control the irreducible clauses are just the pre-solved clauses. Hence, this control will give us, for every clause $\phi$ and every initial solution $V_{\mathcal{X}}$, a pre-solved $\phi$-derivative that has $V_{\mathcal{X}}$ as an solution.

We will add this further control only on the non-deterministic rules (PathRel) and $\left(\operatorname{LangDec}_{\Lambda}\right)$, thus restricting the set of instances of these rules that may be applied. We allow only those instances which preserve the valuation $\left(V_{\mathcal{X}}, V_{\mathcal{P}}\right)$. Using our above example, if $V_{\mathcal{P}}$ satisfies

$$
V_{\mathcal{P}}(\alpha)=f \quad \text { and } \quad V_{\mathcal{P}}(\beta)=g
$$

we may apply only that instance of (PathRel) which transforms $\phi$ into $\phi_{1}=$ $\{\alpha$ II $\beta, x \alpha x, x \beta y\}$. Since the choice of the instances depends only on the path valuation, we will call such restricted derivations $V_{\mathcal{P}}$-strict.

It is easy to see that the above restriction will always enforce finiteness of derivations if the initial path valuation $V_{\mathcal{P}}$ satisfies

$$
V_{\mathcal{P}}(\alpha) \nprec V_{\mathcal{P}}(\beta) \quad \text { where } \quad \alpha \neq \beta \wedge x \alpha y \in \phi \wedge x \beta z \in \phi .
$$

One could say that in this case $V_{\mathcal{P}}$ is prefix free with respect to $\phi$.

For the initial path valuations which are not prefix free we must have a closer look at the (Pre) rule, since this rule is the reason for looping derivations. As (Pre) is a rule which is not $\mathcal{P}$-preserving, the path valuation has to be changed in a $V_{\mathcal{P}}$-strict derivation when (Pre) is applied. This implies that we can yield finiteness of $V_{\mathcal{P}}$-strict derivations if we guarantee that after a finite number of (Pre) applications the initial path valuation has been transformed into a prefix free path valuation.

\footnotetext{
${ }^{3}$ The first example of a looping derivation on page 7 shows that the situation is no different if we add path restrictions.
} 
We will again turn to our example to clarify this. If the initial path valuation $V_{\mathcal{P}}$ for $\phi$ is of the form

$$
V_{\mathcal{P}}(\alpha)=f \quad \text { and } \quad V_{\mathcal{P}}(\beta)=f f f g,
$$

the first rule in a $V_{\mathcal{P}}$-strict $\phi$-derivation could be an application of (PathRel) transforming $\phi=\phi_{0}$ into $\phi_{1}=\{\alpha \dot{\prec} \beta, x \alpha x, x \beta y\}$. Now we are able to apply (Pre), which implies that we have to change $V_{\mathcal{P}}$. Using proposition 6.9 we can use the following $V_{\mathcal{P}}^{\prime}$ :

$$
V_{\mathcal{P}}^{\prime}(\alpha)=f \quad \text { and } \quad V_{\mathcal{P}}^{\prime}(\beta)=f f g .
$$

Proposition 6.9 guarantees that this can be done without loosing $\mathcal{X}$ preservingness. Note that we have shortened $V_{\mathcal{P}}(\beta)$ by $f$. Now we could iterate this twice more before ending up with a prefix free path valuation.

After these remarks we can turn to the technical part.

Theorem 6.12 (Completeness-II) Let $\phi$ be a prime clause, let $\Lambda$ be a set of regular languages which is $\phi$-closed, closed under intersection and decomposition. Then $\mathcal{R}_{\Lambda}$ is $\phi$-complete w.r.t. the first order variables $\mathcal{X}$.

First we need an additional lemma.

Lemma 6.13 There are no infinite derivations using only finitely many instances of (Pre).

Proof. Assume there is such a derivation. Then there exists an infinite sub-derivation not using any instance of (Pre). Let $\phi$ be the starting point of such a derivation. Let $\gamma$ be some clause. Then we define the following functions on $\gamma$ :

$$
\begin{aligned}
\Theta_{1}(\gamma)= & \text { number of concatenations in } \gamma \\
\Theta_{2}(\gamma)= & \text { number of different path variables in } \gamma \\
\Delta^{\phi}(\gamma)= & \text { number of constraints } \alpha \dot{\beta} \beta \text { with } \dot{\diamond} \in\{\dot{\doteq}, \dot{\prec}, \dot{\amalg}\}, \\
& \alpha, \beta \in \mathcal{V} \text { arsp }(\phi) \text { and } \alpha \dot{s} \underline{\text { not in } \gamma} \gamma \\
\Pi(\gamma)= & \text { total number of constraints in } \gamma
\end{aligned}
$$

We define $\Theta(\gamma)$ to be the tuple $\left\langle\Theta_{1}(\gamma), \Theta_{2}(\gamma)\right\rangle$. Using the functions $\Theta, \Delta^{\phi}$ and $\Pi$ we can construct a partial order on clauses by defining $\gamma<_{\phi} \gamma^{\prime}$ iff

$$
\left(\Theta(\gamma)<\Theta\left(\gamma^{\prime}\right)\right)
$$




\begin{tabular}{l|cccc} 
& $\Theta_{1}$ & $\Theta_{2}$ & $\Delta^{\phi}$ & $\Pi$ \\
\hline (PathRel) & $=$ & $=$ & $<$ & \\
(Eq) & $=$ & $<$ & & \\
(LangDec $)$ & $<$ & $=$ & & \\
(Join) & $=$ & $=$ & $=$ & $<$ \\
(Div1) & $<$ & $=$ & & \\
(Div2) & $<$ & $=$ & &
\end{tabular}

Table 1: Monotonicity of the rules w.r.t the measure functions.

or

$$
\left(\Theta(\gamma)=\Theta\left(\gamma^{\prime}\right)\right) \wedge\left(\Delta^{\phi}(\gamma)<\Delta^{\phi}\left(\gamma^{\prime}\right)\right)
$$

or

$$
\left(\Theta(\gamma)=\Theta\left(\gamma^{\prime}\right)\right) \wedge\left(\Delta^{\phi}(\gamma)=\Delta^{\phi}\left(\gamma^{\prime}\right)\right) \wedge\left(\Pi(\gamma)=\Pi\left(\gamma^{\prime}\right)\right)
$$

Here $<$ is the lexicographic ordering on tuples for $\Theta(\gamma)$ and elsewhere the usual numeric comparison. It is easy to check, that $<_{\phi}$ defines a wellfounded, partial ordering on clauses.

Let $\gamma$ be some derivation of $\phi$. Now $\operatorname{Varsp}(\gamma) \subseteq \mathcal{V}$ arsp $(\phi)$ holds, which is important for the value of $\Delta^{\phi}$. In table 1 we have summarized for every nonclash rule other than (Pre) the variation of $\Theta(\gamma), \Delta^{\phi}(\gamma)$ and $\Pi(\gamma)^{4}$. The clash rules are not considered because they automaticly terminate every derivation. The table shows that for every rule $r \gamma \rightarrow_{r} \gamma^{\prime}$ implies $\gamma^{\prime}<_{\phi} \gamma$. Because $<_{\phi}$ is a well-founded ordering and therefore cannot have infinite descending chains, this contradicts our assumption that there is a infinite derivation not using (Pre).

Corollary 6.14 There are no infinite derivations using only finitely many instances of (PathRel).

Proof. By the above lemma we know that there are no infinite derivations without infinite use of (Pre). But (Pre) removes the constraints $\alpha \dot{\prec} \beta$, the existence of which is an application condition for (Pre). But additional constraints of form $\alpha \dot{\prec} \beta$ are only introduced by (PathRel).

Proof of theorem 6.12 (Completeness II). The first condition for $\phi$ completeness was proved in theorem 6.11 (Completeness I). For the second,

\footnotetext{
${ }^{4}$ If a rule decreases the $\Theta$-value, the clause resulting from applying this rule is smaller than the input clause w.r.t $<_{\phi}$ independently of the effects of the rule on the $\Delta^{\phi}$-part. Therefore, we omit the corresponding $\Delta^{\phi}$-entries in this case; and similarly for the П-part.
} 
let $\mathcal{I}$ be some interpretation and $\left(V_{\mathcal{X}}, V_{\mathcal{P}}\right)$ be a valuation with $\left(V_{\mathcal{X}}, V_{\mathcal{P}}\right) \models_{\mathcal{I}} \phi$. We have to show that there is a $\left(\phi, \mathcal{R}_{\Lambda}\right)$-derivative $\gamma$ which is pre-solved and satisfies $\exists V_{\mathcal{P}}^{\prime}:\left(V_{\mathcal{X}}, V_{\mathcal{P}}^{\prime}\right) \models_{\mathcal{I}} \gamma$. This will be done by defining $V_{\mathcal{P}}$-strict derivations, which will always end up in a pre-solved clause. As we have mentioned, we have to redefine the path valuation every time (Pre) is applied. This leads to the following definition: a derivation

$$
\phi=\phi_{0} \rightarrow_{r_{0}} \phi_{1} \cdots \phi_{n} \rightarrow_{r_{n}} \phi_{n+1} \cdots
$$

is called $V_{\mathcal{P}}$-strict if there is a family of path valuations $\left(V_{\mathcal{P}}^{i}\right)$ such that

1. $V_{\mathcal{P}}^{0}=V_{\mathcal{P}}$

2. for each $i$ the proposition $\left(V_{\mathcal{X}}, V_{\mathcal{P}}^{i}\right) \models_{\mathcal{I}} \phi_{i}$ holds; and

3. for each $i$

- $r_{i} \notin\left(\right.$ Pre) implies $V_{\mathcal{P}}^{i}=V_{\mathcal{P}}^{i+1}$ and

- $r_{i}=\frac{\{\alpha \dot{<} \beta, \ldots\} \cup \psi}{\cdots} \in$ (Pre) implies

$$
V_{\mathcal{P}}^{i}={ }_{\mathcal{P}-\{\beta\}} V_{\mathcal{P}}^{i+1} \quad \text { and } \quad V_{\mathcal{P}}^{i}(\beta)=V_{\mathcal{P}}^{i+1}(\alpha) V_{\mathcal{P}}^{i+1}(\beta) .
$$

Now for every $V_{\mathcal{P}}$-strict $\left(\phi, \mathcal{R}_{\Lambda}\right)$-derivation

$$
\phi=\phi_{0} \rightarrow_{r_{0}} \phi_{1} \cdots \phi_{n-1} \rightarrow_{r_{n-1}} \phi_{n}
$$

where $\phi_{n}$ is not pre-solved, there is a $V_{\mathcal{P}}$-strict continuation, as the following argumentation shows. If $\phi_{n}$ is not pre-solved, then there is (by theorem 6.11) a rule which is applicable. We have to show that there is an applicable rule instance such that a corresponding $V_{\mathcal{P}}^{n+1}$ can be found.

If the applicable rule is different from (Pre), then we know that there is an appropriate path valuation $V_{\mathcal{P}}^{n+1}$, as all rules different from (Pre) are either $\mathcal{X} \cup \mathcal{P}$-preserving or globally $\mathcal{X} \cup \mathcal{P}$-preserving. If (Pre) is applicable, then proposition 6.9 shows that we can find an appropriate $V_{\mathcal{P}}^{n+1}$.

Next we must show that there is no infinite $V_{\mathcal{P}}$-strict $\left(\phi, \mathcal{R}_{\Lambda}\right)$-derivation, which finally proves the lemma. This is done by introducing a norm on path valuations. For a path valuation $V_{\mathcal{P}}$ we define $\left|V_{\mathcal{P}}\right|_{\phi}$ to be:

$$
\left|V_{\mathcal{P}}\right|_{\phi}=\sum_{\alpha \in \mathcal{V}_{\operatorname{ars} \mathcal{P}_{\mathcal{P}}(\phi)}}\left|V_{\mathcal{P}}(\alpha)\right|
$$

Now let

$$
\phi_{i} \rightarrow_{r_{i}} \phi_{i+1}
$$


be a step in some $V_{\mathcal{P}}$-strict $\left(\phi, \mathcal{R}_{\Lambda}\right)$-derivation and let $V_{\mathcal{P}}^{i}, V_{\mathcal{P}}^{i+1}$ be the corresponding path valuations. If $r_{i} \notin$ (Pre) we know that $V_{\mathcal{P}}^{i}=V_{\mathcal{P}}^{i+1}$ and hence $\left|V_{\mathcal{P}}^{i}\right|_{\phi}=\left|V_{\mathcal{P}}^{i+1}\right|_{\phi}$. If $r_{i} \in$ (Pre) we know by the third condition of $V_{\mathcal{P}}$-strictness that there are $\alpha$ and $\beta$ such that

$$
V_{\mathcal{P}}^{i}={ }_{\mathcal{P}-\{\beta\}} V_{\mathcal{P}}^{i+1} \quad \text { and } \quad V_{\mathcal{P}}^{i}(\beta)=V_{\mathcal{P}}^{i+1}(\alpha) V_{\mathcal{P}}^{i+1}(\beta) .
$$

$\operatorname{As} \operatorname{Varsp}\left(\phi_{i+1}\right) \subseteq \operatorname{Varsp}\left(\phi_{i}\right) \subseteq \operatorname{Varsp}(\phi)$ this implies $\left|V_{\mathcal{P}}^{i+1}\right|_{\phi}<\left|V_{\mathcal{P}}^{i}\right|_{\phi}$.

As there are no infinite derivations without infinite use of (Pre) this proves that there are no infinite $V_{\mathcal{P}}$-strict derivations.

\subsection{Quasi-Termination}

Lemma 6.15 Let $\phi$ be a prime clause and $\Lambda$ be a finite $\phi$-closed set of regular languages. Then the set of all $\left(\phi, \mathcal{R}_{\Lambda}\right)$-derivatives is finite.

Proof. We will first consider the sets $\mathcal{C}$ which contains every atomic constraint that occur in at least one $\left(\phi, \mathcal{R}_{\Lambda}\right)$-derivative. $\mathcal{C}$ could be seen as the union of all $\left(\phi, \mathcal{R}_{\Lambda}\right)$-derivatives. We will show that $\mathcal{C}$ is finite. As every $\left(\phi, \mathcal{R}_{\Lambda}\right)$-derivative is a subset of $\mathcal{C}$ this will prove the lemma.

First we know that no rule adds new variables. This implies that there are at most $n_{1}=|\mathcal{V} \operatorname{arsp}(\phi)|+|\mathcal{V} \operatorname{arsp}(\phi)|^{2}$ many different path terms. By lemma 6.1 we know that $\Lambda$ is $\gamma$-closed for every $\left(\phi, \mathcal{R}_{\Lambda}\right)$-derivative, which implies that at most $|\Lambda|$ different regular languages are used in the $\left(\phi, \mathcal{R}_{\Lambda}\right)$ derivatives.

Therefore $\mathcal{C}$ contains at most $\mid \mathcal{V}$ ars $\left.\mathcal{X}(\phi)\right|^{2}$ node agreements, $\mid \mathcal{V}$ ars $s_{\mathcal{X}}(\phi) \mid *$ $\left|\operatorname{Varsp}_{\mathcal{P}}(\phi)\right| *\left|\operatorname{Vars}_{\mathcal{X}}(\phi)\right|$ subterm agreements, $n_{1}^{2}$ path divergence constraints, $|\operatorname{Varsp}(\phi)|^{2}$ prefix and equality constraints and $n_{1} *|\Lambda|$ path restriction constraints. Since no rule adds new sort symbols we know that $\mathcal{C}$ contains at most $n_{2} * \mid \mathcal{V}$ ars $s_{\mathcal{X}}(\phi) \mid$ different node restrictions, where $n_{2}$ is the number of sort symbols in $\phi$.

Theorem 6.16 For every prime clause $\phi$ there exists a set of regular languages $\Lambda$ such that $\mathcal{R}_{\Lambda}$ is $\phi$-complete w.r.t. $\mathcal{X}$ and the set $\operatorname{Pre-Solved}\left(\phi, \mathcal{R}_{\Lambda}\right)$ is finite and computable.

Proof. Let reg $(\phi)$ be the set of regular languages used in $\phi$. By lemma 6.2 there must be a finite $\Lambda$ such that $\Lambda$ is $\phi$-closed, closed under intersection and decomposition. Then $\mathcal{R}_{\Lambda}$ is $\phi$-complete w.r.t. $\mathcal{X}$ by theorem 6.12. By lemma 6.15 we know that Pre-Solved $\left(\phi, \mathcal{R}_{\Lambda}\right)$ must be finite. Hence, it suffices to prove that the set Pre-Solved $\left(\phi, \mathcal{R}_{\Lambda}\right)$ is computable. 
To do this we will consider loop-free derivations. A derivation is called loop-free if it is not of the form

$$
\phi_{0} \rightarrow_{r_{1}} \ldots \rightarrow_{r_{i}} \phi_{i} \ldots \rightarrow_{r_{k}} \phi_{k} \ldots
$$

where $\phi_{i}=\phi_{k}$. In order to generate the set of derivatives (or a subset of them) it is enough to consider loop-free derivations. This is because for every pair $\gamma, \gamma^{\prime}$ every $\gamma$-derivation which yields $\gamma^{\prime}$ and is not loop-free can be replaced with a shorter derivation by removing some loop. Iterating this step finally yields a loop-free $\gamma$-derivation for $\gamma^{\prime}$.

Furthermore, the set of all loop-free $\left(\phi, \mathcal{R}_{\Lambda}\right)$-derivations must be finite since $\mathcal{R}_{\Lambda}$ can only generate finitely many $\left(\phi, \mathcal{R}_{\Lambda}\right)$-derivatives by lemma 6.15 , and there are only finitely many rules of $\mathcal{R}$ applicable on every $\left(\phi, \mathcal{R}_{\Lambda}\right)$-derivative. But as we have mentioned we need to consider only the loop-free derivations, which shows that Pre-Solved $\left(\phi, \mathcal{R}_{\Lambda}\right)$ is computable.

Corollary 6.17 For every prime clause $\phi$ there exists a finite and computable set of pre-solved clauses $\Gamma$ such that $\Gamma$ is equivalent to $\phi$.

Proof. Follows from the last theorem and the fact, that every rule is at least $V_{\mathcal{X}}$-sound.

\section{The Second Phase: Satisfiability of Pre-Solved Clauses}

In this section we will present a rule system that transforms each pre-solved clause into an equivalent set of solved clauses, which are satisfiable by lemma 5.3.

We will first make a minor redefinition of divergence. We say that two paths $u, v$ are directly diverging (written $u \mathrm{I}_{0} v$ ) if there are features $f \neq g$ such that $u \in f \mathcal{F}^{*}$ and $v \in g \mathcal{F}^{*}$. Then $u$ II $v$ holds if there are a possible empty prefix $w$ and paths $u^{\prime}, v^{\prime}$ such that $u=w u^{\prime}$ and $v=w v^{\prime}$ and $u^{\prime} \mathrm{I}_{0} v^{\prime}$. Using this definition of divergence and the additional atomic constraint

\section{$\alpha \dot{\mathrm{I}}_{0} \beta \quad$ direct divergence,}

we can (non-deterministically) transform a clause $\phi=\left\{\alpha_{1}\right.$ İ $\left.\alpha_{2}\right\} \cup \psi$ into either $\left\{\alpha_{1} \dot{\mathrm{I}}_{0} \alpha_{2}\right\} \cup \psi$ or $\left\{\alpha_{1} \doteq \beta \cdot \alpha_{1}^{\prime}, \alpha_{2} \doteq \beta \cdot \alpha_{2}^{\prime}, \alpha_{1}^{\prime} \dot{\mathrm{I}}_{0} \alpha_{2}^{\prime}\right\} \cup \psi \cdot{ }^{5}$ By

\footnotetext{
${ }^{5}$ The first case is needed because we do not allow values of path variables to be empty paths.
} 
the definition of $\mathrm{I}_{0}$ we can reduce (non-deterministically) the constraints of form $\alpha_{1} \dot{\mathrm{I}}_{0} \alpha_{2}$ into $\left\{\alpha_{1} \dot{\in} f \mathcal{F}^{*}, \alpha_{2} \dot{\in} g \mathcal{F}^{*}\right\}$ with $f \neq g$. The aim is to process all divergence constraints this way in order to achieve a solved clause.

But we have to reformulate the reduction of divergence constraints. The reason is that we have to evaluate constraints of the form $\alpha_{1} \doteq \beta \cdot \alpha_{1}^{\prime}$. This can produce constraints of the forms $\alpha \cdot \beta \dot{\epsilon} L$ and $\alpha \cdot \beta$ II $\beta^{\prime}$. The second is problematic as we must guess the relation between $\alpha$ and $\beta^{\prime}$. This complicates the termination proof.

We will avoid this problem by using a special property of pre-solved clauses, namely that $\alpha \dot{\text { II }} \beta$ is in a pre-solved clause $\phi$ iff $x \alpha y$ and $x \beta z$ are in $\phi$. Hence, if $\alpha$ II $\beta$ and $\beta$ II $\delta$ are in $\phi$, then $\alpha$ II $\delta$ is also in $\phi$. This implies that we can write $\phi$ as $\dot{\mathrm{II}}\left(A_{1}\right) \uplus \ldots \uplus \dot{\mathrm{I}}\left(A_{n}\right) \uplus \psi$, where $\dot{\mathrm{I}}(A)$ is syntactic sugar for

$$
\left\{\alpha \dot{\amalg} \alpha^{\prime} \mid \alpha \neq \alpha^{\prime} \wedge \alpha, \alpha^{\prime} \in A\right\},
$$

$A_{1}, \ldots, A_{n}$ are disjoint sets of path variables and $\psi$ contains no divergence constraints. Now given such a constraint II $(A)$, suppose that a whole set of path variables $A_{1} \subseteq A$ diverge with the same prefix. Then we can replace II $\left(A_{1}\right) \subseteq \dot{\mathrm{II}}(A)$ by

$$
A_{1}=\beta \cdot A_{1}^{\prime} \cup \dot{\mathrm{I}}_{0}\left(A_{1}^{\prime}\right),
$$

where $\beta$ is new, $A_{1}^{\prime}=\left\{\alpha_{1}^{\prime}, \ldots, \alpha_{n}^{\prime}\right\}$ is a fresh copy of $A_{1}=\left\{\alpha_{1}, \ldots, \alpha_{n}\right\}$ and $A \doteq \beta \cdot A_{1}^{\prime}$ abbreviates the clause $\left\{\alpha_{1} \doteq \beta \cdot \alpha_{1}^{\prime}, \ldots, \alpha_{n} \doteq \beta \cdot \alpha_{n}^{\prime}\right\}$. $\dot{I I}_{0}(A)$ is defined similarly to $\dot{I}(A)$. Under the additional assumption that the common prefix $\beta$ is maximal, it follows that $\beta \dot{\mathrm{I}} \alpha$ holds for $\alpha \in\left(A \perp A_{1}\right)$. If we consider also the effects of $A_{1} \doteq \beta \cdot \mathcal{A}_{1}^{\prime}$ on the subterm agreements in $\phi$, then we get the following non-deterministic rule:

$$
\begin{aligned}
\text { (Reduce } \left._{1}\right) \quad & \frac{x A_{1} Y_{1} \cup \dot{\mathrm{I}}(A) \cup \psi}{\{x \beta z\} \cup z A_{1}^{\prime} Y_{1} \cup \dot{\mathrm{I}}_{0}\left(A_{1}^{\prime}\right) \cup \dot{\mathrm{I}}\left(\{\beta\} \cup A_{2}\right) \cup \psi^{\prime}} \\
& \text { where } \psi^{\prime}=\psi\left[\alpha_{1} \leftarrow \beta \cdot \alpha_{1}^{\prime}, \ldots, \alpha_{n} \leftarrow \beta \cdot \alpha_{n}^{\prime}\right], A_{1} \uplus A_{2}=A, \\
& \left|A_{1}\right|>1 \text { and } z, \beta \text { new. } A_{1}^{\prime} \text { is a disjoint copy of } A_{1} \cdot x A_{1} Y_{1} \text { is } \\
& \text { short for }\left\{x \alpha_{1} y_{1}, \ldots, x \alpha_{n} y_{n}\right\} \cdot \psi \text { may not contain constraints } \\
& \text { of form } \delta \cdot \delta^{\prime} \dot{\epsilon} L \psi \text { in } \psi
\end{aligned}
$$

Note that we have avoided constraints of the form $\alpha \cdot \beta$ II $\beta^{\prime}$. Additionally, we use the non-deterministic rules 
$\begin{array}{lc}\text { (Reduce }) & \frac{\dot{\mathrm{I}}(A) \cup \psi}{\dot{\mathrm{I}}_{0}(A) \cup \psi} \\ \text { (Solv) } & \frac{\dot{\mathrm{I}}_{0}(A) \cup \psi}{\left\{\alpha \in f_{\alpha} \cdot F^{*} \mid \alpha \in A\right\} \cup \psi} \quad f_{\alpha} \neq f_{\alpha^{\prime}} \text { for } \alpha \neq \alpha^{\prime} .\end{array}$

( Reduce $_{2}$ ) is needed as path variables always denote non-empty paths. We will see (Reduce 1$)$ and (Reduce 2 ) as one single rule (Reduce). To complete our rule system, we need the rules $\left(\operatorname{LangDec}_{\Lambda}\right)$, (DecClash), (Join) and (Empty). Since we will show that the rule system is terminating, we can replace ( $\operatorname{LangDec}_{\Lambda}$ ) by a simpler version, namely

$$
\begin{aligned}
\text { (LangDec dfun } \left._{\text {f }}\right) & \frac{\{\alpha \cdot \beta \dot{\epsilon} L\} \cup \psi}{\{\alpha \dot{\epsilon} P\} \cup\{\beta \dot{\epsilon} S\} \cup \psi} \quad P \cdot S \subseteq L, \quad(P, S) \in \operatorname{dfun}(L) \\
& L \text { must contain a path } w \text { with }|w|>1 .
\end{aligned}
$$

Here dfun $: \mathcal{P}\left(\mathcal{F}^{+}\right) \rightarrow \mathcal{P}\left(\mathcal{F}^{+}\right) \times \mathcal{P}\left(\mathcal{F}^{+}\right)$is a decomposition function that assigns to each regular language $L$ a finite set of decompositions. dfun is called decomposition complete if for every regular language $L$ and every path $w=w_{1} w_{2} \in L$ there is a pair $(P, S)$ in dfun $(L)$ with $w_{1} \in P$ and $w_{2} \in S$. The complete set of rules is denoted $\mathcal{R}_{\text {dfun }}^{\text {Solv }}$.

After the explanation of the rule system we can commence the technical part. Since we have added constraints of the form $\alpha \dot{\mathrm{I}}_{0} \beta$, we have to extend condition 5 in the definition of a solved clause as presented on page 13 . We require solved clauses not to contain constraints of the form $\alpha \dot{\mathrm{I}}_{0} \beta$.

A clause $\phi$ is called partitioned if the set of divergence constraints of $\phi$ is of the form II $\left(A_{1}\right) \biguplus \ldots \uplus \dot{\mathrm{I}}\left(A_{k}\right) \biguplus \dot{\mathrm{I}}_{0}\left(A_{k+1}\right) \uplus \ldots \biguplus \dot{\mathrm{I}}_{0}\left(A_{n}\right)$, where the $A_{i}$ are disjoint.

Proposition 7.1 There exists a decomposition function dfun that is decomposition complete.

Proof. See proof of lemma 6.2 for the construction of such a function.

Proposition 7.2 Let $\phi$ be a pre-solved clause and let $\gamma$ be a $\left(\phi, \mathcal{R}_{\mathrm{dfun}}^{\text {Solv }}\right)$ derivative. Then $\gamma$ is partitioned. Furthermore, for every pair of variables $\alpha, \beta$ such that $\alpha \neq \beta, x \alpha y \in \gamma$ and $x \beta z \in \gamma$ we have $\gamma \models \alpha$ II $\beta$. 
Proposition 7.3 For every partitioned clause $\phi$ the rule (Reduce) $=\left(\right.$ Reduce $\left._{1}\right)+\left(\right.$ Reduce $\left._{2}\right)$ is Vars $\mathcal{X}(\phi)$-sound and globally Vars $(\phi)-$ preserving. The rule (Solv) is $V_{\mathcal{X}} \cup V_{\mathcal{P}}$-sound and $V_{\mathcal{X}} \cup V_{\mathcal{P}}$-preserving. If dfun is decomposition complete, then (LangDec $c_{\mathrm{dfun}}$ ) is $V_{\mathcal{X}} \cup V_{\mathcal{P}}$-sound and $V_{\mathcal{X}} \cup V_{\mathcal{P}}$-preserving.

Lemma $7.4 \mathcal{R}_{\mathrm{dfun}}^{\text {Solv }}$ is terminating.

Proof. For (Solv), (Join), (LangDec), (DecClash) and (Empty) it is trivial to see that there are no infinite derivations using only these rules. Furthermore, there are no derivations which use (Reduce) infinitely often, since during every application of (Reduce) at least one divergence constraint is removed (note that $\left|A_{1}\right|>1$ is an application condition of (Reduce 1 )). Hence, there are no infinite $\mathcal{R}_{\text {dfun }}^{\text {Solv }}$-derivations.

Lemma 7.5 Let $\phi$ be a pre-solved clause. If dfun is decomposition complete, then a $\left(\phi, \mathcal{R}_{\mathrm{dfun}}^{\text {Solv }}\right)$-derivative is $\mathcal{R}_{\mathrm{dfun}}^{\text {Solv }}$-irreducible if and only if it is solved.

Proof. Let $\gamma$ be a $\left(\phi, \mathcal{R}_{\text {dfun }}^{\text {Solv }}\right)$-derivative. We have to show that if $\gamma$ is not solved, then one of the rules applies. We will check all conditions that are stated in the definition on page 13.

Condition 1 is satisfied by every $\left(\phi, \mathcal{R}_{\mathrm{dfun}}^{\text {Solv }}\right)$-derivative since $\phi$ is pre-solved and we do not add or change any sort restriction constraint. If one of the conditions 2 or 3 is not satisfied, then one of the rules (Join) or (Empty) will apply. Condition 6 is satisfied by every $\left(\phi, \mathcal{R}_{\text {dfun }}^{\text {Solv }}\right)$-derivative by proposition 7.2. Now let's check the conditions 4 and 5:

$\gamma$ contains a constraint $\alpha \cdot \beta \dot{\epsilon} L$. (LangDec dfun) or (DecClash) is applicable.

$\gamma$ contains a constraint $\alpha \dot{\mathrm{I}}_{0} \beta$. Then $\gamma$ is of the form $\dot{\mathrm{II}}_{0}(A) \cup \psi$ by proposition 7.2, which implies that (Solv) is applicable.

$\gamma$ contains a constraint $\alpha \dot{\mathrm{I}} \beta$. By proposition 7.2 we know that in this case $\gamma$ is of the form $\dot{I I}(A) \cup \psi$. Given the above we can assume that (Reduce) is applicable.

Lemma 7.6 For every pre-solved clause $\phi$ there is a finite and effectively computable set of solved clauses $\Gamma$ such that for every $\mathcal{I}$

$$
\left.\llbracket \phi \rrbracket \rrbracket_{\mathcal{V}_{\text {ars }}(\phi)}^{\mathcal{I}}=\bigcup_{\gamma \in \Gamma} \llbracket \gamma\right]_{\mathcal{V}_{\text {Vars }}^{\mathcal{I}}(\phi)} .
$$


Proof. Follows from propositions 7.1, 7.2 and 7.3 and lemmas 7.4 and 7.5 .

Corollary 7.7 Satisfiability of pre-solved clauses is decidable.

Finally, we are able to combine both phases.

Theorem 7.8 Satisfiability of prime clauses is decidable.

Proof. Follows from the corollaries 6.17 and 7.7 .

\section{Conclusion}

We have shown that the pure existential fragment of feature logic extended by regular path expressions is decidable. The main prerequisite for achieving this result was to switch from the original, unsorted syntax to a two-sorted syntax. For each clause in the original syntax we get an equivalent clause in the new syntax by translating a regular path expression $x L y$ into $\{x \alpha y, \alpha \dot{\epsilon}$ $L\}$ with $\alpha$ new.

The result of the translation constitutes a special class of clauses: the class of prime clauses. The main restriction imposed on prime clauses is that for each path variable $\alpha$ there is at most one constraint $x \alpha y$ contained in a clause. For prime clauses we have presented an algorithm that transforms a clause into an equivalent set of pre-solved clauses. In a second phase pre-solved clauses are checked for satisfiability by transforming them into an equivalent set of solved clauses. Since every solved clause is prime, the result may be reused for later computation.

Our syntax is more expressive than the original one. Although restriction to prime clauses was sufficient for our purposes, it may be interesting to examine whether decidability can be preserved in the absence of the restriction.

\section{Acknowledgements}

I would like to thank Jochen Dörre, Joachim Niehren, Stephen Spackman and Ralf Treinen for helpful discussions and reading draft versions of the paper. In particular, I am grateful to Joachim Niehren for his comments on an earlier draft.

The research reported in this paper has been supported by the Bundesministerium für Forschung und Technologie under contract ITW 90020 (DISCO). 


\section{References}

[AK86] Hassan Aït-Kaci. An algebraic semantics approach to the effective resolution of type equations. Theoretical Computer Science, 45:293-351, 1986.

[AKLN87] Hassan Aït-Kaci, Patrick Lincoln, and Roger Nasr. Le Fun: Logic, equations, and functions. In Proceedings of the 1987 Symposium on Logic Programming, pages 17-23. IEEE Computer Society, 1987.

[AKN86] Hassan Ait-Kaci and Roger Nasr. Login: A logic programming language with built-in inheritance. The Journal of Logic Programming, 3:185-215, 1986.

[AKP91] Hassan Aït-Kaci and Andreas Podelski. Towards a meaning of LIFE. In Proc. of the PLILP'91, Springer LNCS vol. 528, pages 255-274. Springer-Verlag, 1991.

[AKPS92a] H. Aït-Kaci, A. Podelski, and G. Smolka. A feature-based constraint system for logic programming with entailment. In Fifth Generation Computer Systems 1992, pages 1012-1021, Tokyo, Japan, June 1992. Institute for New Generation Computer Technology.

[AKPS92b] Hassan Aït-Kaci, Andreas Podelski, and Gert Smolka. A feature-based constraint system for logic programming with entailment. In Fifth Generation Computer Systems 1992, pages 1012-1021, Tokyo, Japan, June 1992. Institute for New Generation Computer Technology.

$\left[\mathrm{BBN}^{+} 91\right]$ Franz Baader, Hans-Jürgen Bürckert, Berhard Nebel, Werner Nutt, and Gert Smolka. On the expressivity of feature logics with negation, functional uncertainity, and sort equations. Research Report RR-91-01, DFKI, Postfach 2080, 6750 Kaiserslautern, Germany, 1991.

[BS93] Rolf Backofen and Gert Smolka. A complete and recursive feature theory. In Proc. of the $31^{\text {th }}$ ACL, Columbus, Ohio, 1993. To appear. Full version has appeared as Research Report RR92-30, DFKI, Stuhlsatzenhausweg 3, 6600 Saarbrïcken 11, Germany.

[Der87] Nachum Dershowitz. Termination of rewriting. Journal of Symbolic Computation, 3:69-116, 1987. 
[Joh88] Mark Johnson. Attribute-Value Logic and the Theory of Grammar, volume 16 of CSLI Lecture Notes. CSLI, 1988.

[Joh91] M. Johnson. Logic and feature structures. In Proceedings of IJCAI-91, Sydney, Australia, 1991.

[KB82] Ronald M. Kaplan and Joan Bresnan. Lexical-Functional Grammar: A formal system for grammatical representation. In J. Bresnan, editor, The Mental Representation of Grammatical Relations, pages 173-381. MIT Press, Cambridge (MA), 1982.

[Kel91] Bill Keller. Feature logics, infinitary descriptions and the logical treatment of grammar. Cognitive Science Research Report 205, Univerity of Sussex, School of Cognitive and Computing Sciences, 1991.

[KM88] R. M. Kaplan and J. T. Maxwell III. An algorithm for functional uncertainty. In Proceedings of the 12th International Conference on Computational Linguistics, pages 297-302, Budapest, Hungary, 1988.

[KR86] Robert T. Kasper and William C. Rounds. A logical semantics for feature structures. In Proceedings of the 24th Annual Meeting of the ACL, Columbia University, pages 257-265, New York, N.Y., 1986.

[KZ88] Ronald M. Kaplan and Annie Zaenen. Long-distance dependencies, constituent structure, and functional uncertainty. In M. Baltin and A. Kroch, editors, Alternative Conceptions of Phrase Structure. University of Chicago Press, Chicago, 1988.

[RK86] William C. Rounds and Robert Kasper. A complete logical calculus for record structures representing linguistic information. In Proc. of the Symposium on Logic in Computer Sciences, pages 38-43, Cambridge (MA), 1986. IEEE Computer Society.

[Shi86] Stuart M. Shieber. An Introduction to Unification-Based Approaches to Grammar, volume 4 of CSLI Lecture Notes. Stanford University, Stanford (CA), 1986.

[Smo88] Gert Smolka. A feature logic with subsorts. LILOG-Report 33, IWBS, IBM Deutschland, Stuttgart, May 1988.

[Smo92] Gert Smolka. Feature constraint logics for unification grammars. Journal of Logic Programming, 12:51-87, 1992. 
[ST92] Gert Smolka and Ralf Treinen. Records for logic programming. In Krzysztof Apt, editor, Proceedings of the Joint International Conference and Symposium on Logic Programming, pages 240-254, Washington, USA, 1992. The MIT Press. Full version has appeared as Research Report RR-92-23, DFKI, Stuhlsatzenhausweg 3, 6600 Saarbrücken 11, Germany. 